\title{
A telescope detection system for direct and high resolution spectrometry of intense neutron fields
}

\author{
S. Agosteo a, b , A. Fazzi a, b, M.V. Introini a, b , M. Lorenzoli a, b , A. Pola ${ }^{\text {a, b, * }}$ \\ a Politecnico di Milano, Dipartimento di Energia, Sezione di Ingegneria Nucleare, Via Lambruschini 4, 20156 Milano, Italy \\ ${ }^{\mathrm{b}}$ INFN, Sezione di Milano, Via Celoria 16, 20133 Milano, Italy
}

\section{H I G H L I G H T S}

- An innovative neutron spectrometer based on a monolithic telescope is proposed.

- The device exploits an active-converter.

- The geometrical configuration allows to have a high resolution ( $<250 \mathrm{keV}-\mathrm{FWHM})$.

- The system demonstrates to perform a direct measurement of neutron spectra.

\section{A R T I C L E I N F O}

\section{Article history:}

Received 26 August 2015

Received in revised form

27 November 2015

Accepted 7 December 2015

Available online 12 December 2015

\section{Keywords:}

Neutron spectrometer

Silicon detector

High resolution neutron spectrometry

\begin{abstract}
A B S T R A C T
A high energy- and spatial-resolution telescope detector was designed and constructed for neutron spectrometry of intense neutron fields. The detector is constituted by a plastic scintillator coupled to a monolithic silicon telescope (MST), in turn consisting of a $\Delta \mathrm{E}$ and an $\mathrm{E}$ stage. The scintillator behaves as an "active" recoil-proton converter, since it measures the deposited energy of the recoil-protons generated across. The MST measures the residual energy of recoil-protons downstream of the converter and also discriminates recoil-protons from photons associated to the neutron field. The lay-out of the scintillator/MST system was optimized through an analytical model for selecting the angular range of the scattered protons. The use of unfolding techniques for reconstructing the neutron energy distribution was thus avoided with reasonable uncertainty (about $1.6 \%$ in neutron energy) and efficiency (of the order of $10^{-6}$ counts per unit neutron fluence). A semi-empirical procedure was also developed for correcting the non-linearity in light emission from the organic scintillator. The spectrometer was characterized with quasi-monoenergetic and continuous fields of neutrons generated at the $\mathrm{CN}$ Van De Graaff accelerator of the INFN-Legnaro National Laboratory, Italy, showing satisfactory agreement with literature data.
\end{abstract}

๑) 2015 Elsevier Ltd. All rights reserved.

\section{Introduction}

Neutron spectrometers (Brooks and Klein, 2002) can be classified against the principle exploited for assessing the energy distribution of a neutron field, i.e.: i) measurement of the energy of charged particles released in neutron-induced nuclear reactions; ii) measurement of the neutron velocity; iii) threshold-reactions, in which a minimum neutron energy is indicated by the appearance of a neutron-induced effect such as radioactivity, a specific gammaray energy or a phase transition; iv) experimental data unfolding

\footnotetext{
* Corresponding author. Politecnico di Milano, Dipartimento di Energia, Sezione di Ingegneria Nucleare, Via Lambruschini 4, 20156 Milano, Italy.

E-mail address: andrea.pola@polimi.it (A. Pola).
}

from a set of detectors showing a different response to neutron energy; v) measurement of the energy of recoil nuclei from neutron elastic scattering.

Neutron energy is assessed with the time-of-flight technique by measuring the neutron flight time over a known distance. A first method requires the neutron is scattered in a start detector, e.g., an organic scintillator, and the time-of-flight to a downstream detector placed at a known distance and angle is measured (Elevant et al., 1995; Manduchi et al., 1995; Kurosawa et al., 1999; Meigo et al., 1999; Elevant, 2002). A second method uses a start signal provided by an associated particle or quantum that is emitted from the neutron source at the same time as the neutron is (Böttger et al., 1990; Colonna and Tagliente, 1998).

The Bonner sphere spectrometer (BSS, Bramblett et al., 1960) consists of a set of moderating spheres of different diameter 
housing a thermal neutron detector at their center. The spherical shape of the moderator allows approaching an isotropic angular response. Fast neutrons irradiating the spheres are slowed-down to thermal energies with a different effectiveness, depending on the moderator dimensions and on the neutron energy. For a sphere of a given diameter, the response represents the number of events acquired by the thermal neutron detector per unit fluence of neutrons of a given energy impinging on the moderator. The response variation versus neutron energy is the response function of a sphere of a given dimension. The set of response functions of all the spheres of a BSS constitutes its response matrix. Recently, a novel active neutron spectrometer condensing the functionality of Bonner spheres in a single moderator was also proposed (Bedogni et al., 2014).

Recoil spectrometers are either detectors in which recoils at all angles to the incident neutron direction are accepted for measurement, or recoil telescopes, in which recoils at a particular angle (preferably $0^{\circ}$ ) are selected for analysis. The spectrometer response function (pulse height spectrum of secondary particles resulting from monoenergetic neutron irradiation) is typically a broad continuum for the former category and a narrow function for the latter one. Recoil spectrometers based on proportional counters are widely used in the energy range $50 \mathrm{keV}$ to a few $\mathrm{MeV}$ (Ing et al., 1997; Rosenstock et al., 1997; Pichenot et al., 2002).

Although ${ }^{4} \mathrm{He}$ recoil devices can extend the energy range up to about $15 \mathrm{MeV}$, organic scintillators are usually the preferred detectors for spectrometry at higher energies (Harvey and Hill, 1979; Klein and Neumann, 2002), in particular stilbene crystals and liquid scintillators, due to their capability of discriminating between neutron and photon events by pulse-shape analysis. The response functions of these detectors are dominated by $n-p$ elastic scattering in this energy range. Since the $n-p$ cross-section is wellknown, response matrices and detection efficiencies can be computed accurately and neutron spectra can be reliably unfolded from measured pulse height spectra. Charged particles produced in the scintillator by neutron interactions with carbon nuclei make significant contributions to the response functions of organic scintillators at incident neutron energies above about $8 \mathrm{MeV}$, but are adequately accounted for in simulated response functions for energies up to about $15 \mathrm{MeV}$ (Klein and Neumann, 2002).

Recoil telescope spectrometers strive to achieve a simple response function, ideally a single sharp peak at a pulse height uniquely related to the neutron energy. A wide variety of detection systems have been designed for approaching this requirement. Recoil telescopes can provide simple response functions but usually with low neutron detection efficiency, typically $<0.01 \%$. Another approach that is used to achieve a simple response function is the capture-gated neutron spectrometer (Aleksan et al., 1989; Kamykowski, 1992; Aoyama et al., 1993; Bart Czirr, 1994; Bertin et al., 1994). This is a recoil detector spectrometer, usually a liquid or plastic scintillator, which selects events in which neutrons transfer all of their energy by elastic and inelastic scattering within the scintillator. A delayed coincidence between the summed pulse height signal and the subsequent $(0.2-50 \mathrm{~ms})$ signal due to capture of the neutron after moderation to a low energy $(<10 \mathrm{eV})$ is required. Neutron capture is detected by doping the organic scintillator (liquid or plastic) with ${ }^{10} \mathrm{~B}$ or ${ }^{6} \mathrm{Li}$, or by incorporating a separate low-energy neutron detector in the system. The neutron energy is obtained from the summed pulse height signals which in general generate a broad distribution (FWHM 50\%). However, detection efficiencies of about $10 \%$ can be achieved.

A monolithic silicon telescope coupled to a polyethylene converter was studied by Agosteo et al., 2007, 2011, as a recoil-proton spectrometer for low-energy neutron fields (below about $8 \mathrm{MeV}$ ), with an efficiency of the order of $10^{-4}$ counts per unit neutron fluence. The device consists of a surface $\Delta \mathrm{E}$ stage, about $2 \mu \mathrm{m}$ in thickness, and an E stage, $500 \mu \mathrm{m}$ in thickness, made out of a single silicon wafer. The sensitive area of the detector is about $1 \mathrm{~mm}^{2}$. The two stages share a deep $\mathrm{p}^{+}$electrode obtained through an highenergy boron implantation (Tudisco et al., 1999).

The neutron spectrometer is assembled by placing a $1 \mathrm{~mm}$ thick polyethylene layer adjacent to the $\Delta \mathrm{E}$ stage of the monolithic silicon telescope. This device accomplishes the role of detecting the recoilprotons generated in the converter by neutrons impinging on hydrogen nuclei. The spectra acquired by the silicon detector correspond to the distribution of energy deposited within the sensitive volume by recoil-protons only (apart from the secondary electrons generated by background photons interacting with the detector assembly).

Since the $\Delta \mathrm{E}$ stage measures a LET-related quantity, the acquisition of the time-correlated distribution of events which deposit an energy $\Delta \mathrm{E}$ in the $\Delta \mathrm{E}$ stage and a total energy $\mathrm{E}_{\mathrm{MST}}$ in the whole detector (the so called $\Delta \mathrm{E}-\mathrm{E}_{\mathrm{MST}}$ scatter plot) allows a particlerelated event discrimination.

The energy distributions of the neutron yield are reconstructed with an unfolding algorithm based on a non-linear least-squares method. The response matrix was calculated by using an analytical model developed by Agosteo and Pola, 2008.

The aim of the present work is to develop a compact device capable of performing real-time direct neutron spectrometry with high energy resolution. It should be noted that the instruments described above do not meet this requirement, apart from TOF detectors, which are based on very complex detection systems. On the other hand, the experimental data acquired with multisphere and recoil nuclei devices must be unfolded through dedicated algorithms which: i) limit the simplicity of the systems, ii) deteriorate their energy resolution and iii) prevent the possibility of a real-time measurement of neutron spectra.

The spectrometer proposed in the present work is composed by a plastic scintillator coupled to a photomultiplier and a residual energy stage constituted by a monolithic silicon telescope (MST). The scintillator behaves as an "active" recoil-proton converter, while the silicon telescope measures the residual energy of recoilprotons downstream of the converter. Moreover, the MST discriminates the recoil-protons from photons associated to the neutron field.

\section{Spectrometer design}

As discussed in the Introduction, the spectrometer proposed herein consists of a monolithic silicon telescope (MST) coupled to a plastic scintillator acting as an active recoil-proton converter. Agosteo et al., 2007, 2011 used the same MST, coupled adjacently to a "passive" recoil-proton converter (a polyethylene layer $1 \mathrm{~mm}$ in thickness), for measuring the energy distribution of neutrons from low energy charged particles. A scheme of that device (polyethylene converter telescope, PCT in the following) is shown in Fig. 1. The MST is characterized by a dead layer of titanium (about $0.24 \mu \mathrm{m}$ in thickness), a $\Delta \mathrm{E}$ and a $\mathrm{E}$ stage about $2 \mu \mathrm{m}$ and $500 \mu \mathrm{m}$ in thickness, respectively.

The energy $E_{p}$ transferred to a recoil-proton by a neutron of energy $E_{n}$ through an elastic collision is:

$E_{p}=E_{n} \cdot \cos ^{2}(\theta)$

where $\theta$ is the recoil-proton scattering angle.

In the PCT configuration, the distribution of the residual energy of recoil-protons generated at all scattering angles in the polyethylene converter is measured by the MST. Therefore, any information about $\mathrm{E}_{\mathrm{p}}$ and $\theta$, and therefore $\mathrm{E}_{\mathrm{n}}$, is completely lost and the 


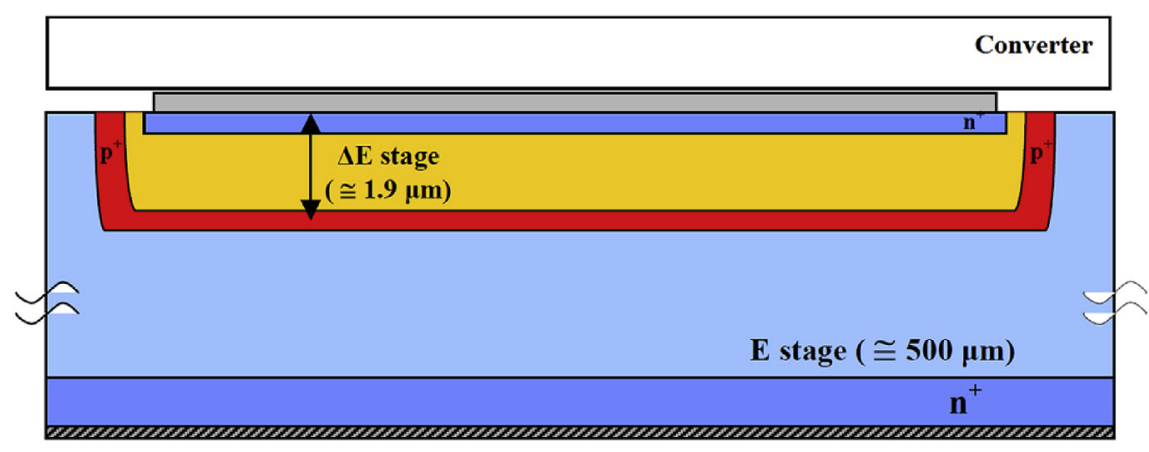

Fig. 1. Scheme of the monolithic silicon telescope coupled to a plastic converter.

experimental spectra of energy deposited in the MST must be unfolded in order to reconstruct the neutron energy distribution.

The actual value of the energy $\mathrm{E}_{\mathrm{p}}$ can be derived by measuring event-by-event the energy lost by recoil-protons in the converter, e.g. through a plastic polyvinyltoluene scintillator replacing the polyethylene layer, but it is not sufficient to have a biunique relation with the energy of the impinging neutron due to the uncertainty in the scattering angle value.

The neutron spectrometer proposed in this work (active converter spectrometer, ACSpect) provides a reduction of the angular distribution of recoil-protons impinging on the MST by separating the scintillator converter from the MST. The distance between the two detectors should be optimized for minimizing the angular range of recoil-protons impinging on the MST without leading to a high decrease in efficiency (which in turn would lead to unacceptably long measurement times). Moreover, this limited angular range should be optimized with respect to the uncertainty in the assessed neutron energy. The spectrometer must be placed in vacuum, in order to avoid that recoil-protons lose their energy (in air) while traversing the distance between the scintillator/converter and the MST. It should be stressed that such a spectrometer is highly directional, i.e. the neutron source should approach a pointsource configuration and therefore it can be applied for measuring the energy distribution of neutrons generated either by accelerated particles on targets or by isotopic sources.

\section{Analytical model}

\subsection{Particle trajectory and energy deposition}

The analytical approach described in the following is based on the energy-range and energy-stopping power relations taken from ICRU 49 (ICRU, 1993) and the SRIM code (Ziegler et al., 1985; Ziegler and Biersack, 2008). Energy straggling was not considered in this model.

The model calculates the energy deposition in the scintillator and in the MST as a function of the recoil-proton trajectory (Fig. 2). It should be noted that for the PCT configuration, the distance distR between the scintillator and the MST is equal to 0 .

In order to be detected by the MST, recoil-protons must leave the scintillator converter, traverse the titanium dead-layer, interact with the $\Delta \mathrm{E}$ stage and, if they possess enough energy, with the $\mathrm{E}$ stage. The chance of reaching the $\Delta \mathrm{E}$ stage depends on both geometry and energy. The model considers a MST with a square crosssection $2 L_{\text {det }}$ in width (i.e $L_{\text {det }}$ is half the detector side).

In order to interact with the $\Delta \mathrm{E}$ stage from a starting position $(x, y, z)$ inside the converter, a recoil-proton must leave the titanium layer with coordinates $\left|X_{\text {det }}\right|$ and $\left|Y_{\text {det }}\right|$ both less than $L_{d e t}$, where:

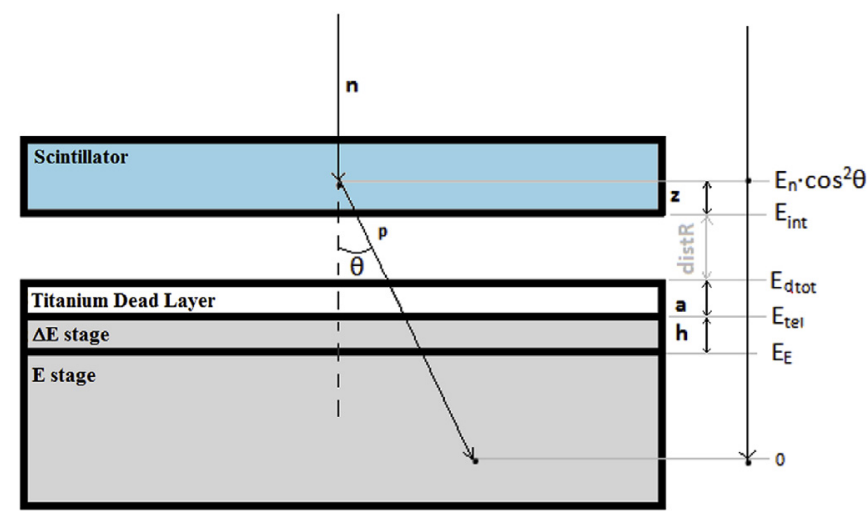

Fig. 2. Sketch of the spectrometer layout.

$X_{\text {det }}=x+(z+\operatorname{distR}+a) \cdot \tan (\theta) \cdot \cos (\varphi)$

$Y_{\text {det }}=y+(z+\operatorname{dist} R+a) \cdot \tan (\theta) \cdot \sin (\varphi)$

where $\theta$ and $\varphi$ are the recoil-proton emission angles and $a$ is the dead layer thickness (Fig. 3).

A recoil-proton of energy $E_{p}=E_{n} \cdot \cos ^{2}(\theta)$ does not stop completely in the scintillator converter if it is set in motion in the converter at a distance $z$ from the exit surface lower than $R^{\text {scint }}\left(E_{p}\right) \times \cos (\theta)$, where $R^{\text {scint }}\left(E_{p}\right)$ is the recoil proton range of initial energy $E_{p}$ in the scintillator material.

The range $R^{s c i n t}\left(E_{\text {int }}\right)$ in the scintillator material of a proton leaving the scintillator with energy $E_{i n t}=E_{p}-E_{\text {scint }}$ (where $E_{\text {scint }}$ is the energy deposited in the converter) is then the difference between its range at the initial energy and its path in the scintillator:

$R^{\text {scint }}\left(E_{\text {int }}\right)=R^{\text {scint }}\left(E_{n} \cdot \cos ^{2}(\theta)\right)-\frac{z}{\cos (\theta)}$

By inverting the range-energy relation, $E_{\text {int }}(\theta, z)$ results to be:

$E_{\text {int }}(\theta, z)=E^{\text {poly }}\left(R^{\text {poly }}\left(E_{n} \cdot \cos ^{2}(\theta)\right)-\frac{z}{\cos (\theta)}\right)$

and, as mentioned above, the energy $E_{\text {scint }}$ deposited in the converter is:

$E_{\text {scint }}(\theta, z)=E_{n} \cdot \cos ^{2}(\theta)-E_{\text {int }}(\theta, z)$

The air volume between the converter and the silicon telescope was considered in the analytical approach, although the whole spectrometer was placed in vacuum. A recoil proton would impinge 


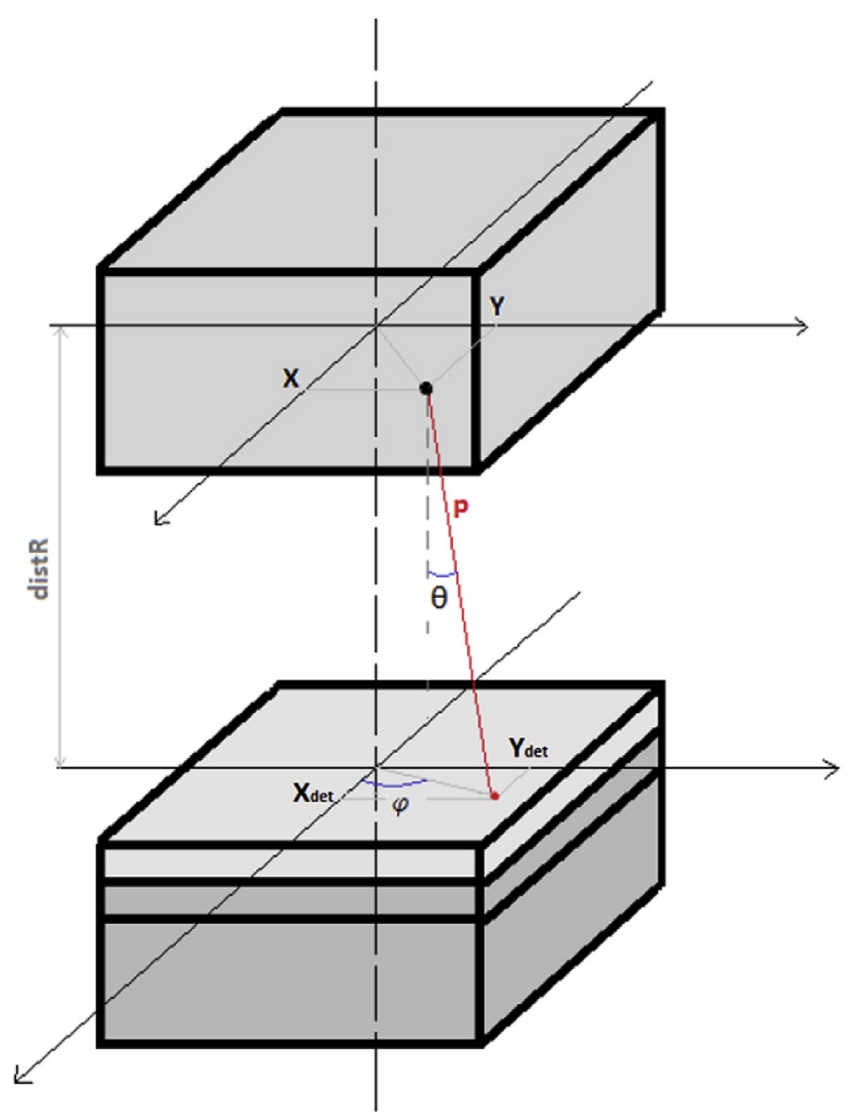

Fig. 3. Three-dimensional scheme of recoil-proton trajectory across the neutron spectrometer.

on the titanium dead layer, if its range in air $R^{A i r}\left(E_{\text {int }}\right)$ is $R^{\text {Air }}\left(E_{\text {int }}\right)>(($ dist $R) /(\cos (\theta)))$; by using the same method adopted of the upstream layer, its residual range in air and energy $E_{d t o t}$ result to be:

$R^{A i r}\left(E_{\text {dtot }}\right)=R^{A i r}\left(E_{\text {int }}(\theta, z)\right)-\frac{\text { dist } R}{\cos (\theta)}$

$E_{d t o t}(\theta, z)=E^{A i r}\left(R^{A i r}\left(E_{\text {int }}(\theta, z)\right)-\frac{d i s t R}{\cos (\theta)}\right)$

respectively.

For being detected by the MST, the recoil-proton must get through the titanium dead layer and release energy inside the $\Delta \mathrm{E}$ stage. Therefore, its range in titanium must be $R^{T i}\left(E_{d t o t}\right)>(a /(\cos (\theta)))$ and its range and energy $E_{t e l}$, while impinging on the $\Delta \mathrm{E}$ stage, result to be:

$R^{T i}\left(E_{\text {tel }}\right)=R^{T i}\left(E_{d t o t}(\theta, z)\right)-\frac{a}{\cos (\theta)}$

$E_{\text {tel }}(\theta, z)=E^{T i}\left(R^{T i}\left(E_{d t o t}(\theta, z)\right)-\frac{a}{\cos (\theta)}\right)$

respectively.

A proton impinging on the $\Delta \mathrm{E}$ stage is detected by the MST, regardless of its capability of interacting also with the $\mathrm{E}$ stage. Its residual range and energy $E_{E}$ downstream of the $\Delta \mathrm{E}$ stage are then:
$R^{S i}\left(E_{E}\right)=R^{S i}\left(E_{t e l}(\theta, z)\right)-\frac{h}{\cos (\theta)}$

$E_{E}(\theta, z)=E^{S i}\left(R^{S i}\left(E_{t e l}(\theta, z)\right)-\frac{h}{\cos (\theta)}\right)$

respectively, where $h$ is the $\Delta \mathrm{E}$ stage thickness.

Equations (11) and (12) are valid for protons crossing the $\Delta \mathrm{E}$ stage ("crossers"). If the recoil-proton range downstream of the dead layer is $R^{S i}\left(E_{t e l}\right) \leq(h /(\cos (\theta)))$, the proton stops completely inside the $\Delta \mathrm{E}$ stage ("stopper") and $E_{E}$ is equal to zero; in both cases, the energy deposited in the $\Delta \mathrm{E}$ stage is:

$E_{\Delta E}(\theta, z)=E_{t e l}(\theta, z)-E_{E}(\theta, z)$.

\subsection{Neutron interaction probability in the converter}

For a quantitative evaluation of the fluence response functions, the probability $\Pi\left(E_{n}\right)$ of generating a recoil-proton in the scintillator/converter per unit neutron fluence should be estimated. This probability should take into account the attenuation of the neutron field inside the scintillator, which increases for decreasing neutron energies.

The probability $\Pi\left(E_{n}\right)$ was evaluated by assuming an exponential attenuation of the neutron fluence inside the converter and by neglecting multiple neutron scattering. By integrating the probability of undergoing an interaction with the hydrogen nuclei per unit path length over the layer of interest:

$\Pi\left(E_{n}\right)=A \cdot \int_{l-R_{\text {scint }}\left(E_{n}\right)}^{l} e^{-\Sigma_{\text {tot }} \cdot x} \cdot \Sigma_{H} \cdot d x$

where $A$ is the detector sensitive area, $l$ is the scintillator thickness $(2 \mathrm{~mm}), \Sigma_{H}$ is the macroscopic cross section of hydrogen, $\Sigma_{\text {tot }}$ is the total macroscopic cross section (hydrogen plus carbon) at the energy $E_{n}$ and $R^{\text {scint }}\left(E_{n}\right)$ is the maximum range in the scintillator of recoil-protons. It should be mentioned that a polyvinyltoluene converter was accounted for in the Monte Carlo simulations described in the following Section.

\section{Monte Carlo simulations}

In order to have a simple and time-effective code for calculating the response functions of the spectrometer and studying the best experimental configuration, a dedicated Monte Carlo algorithm based on the analytical model described in Section 3 was developed. This algorithm samples the neutron energy from a given source distribution (e.g. monoenergetic), by taking into account the neutron scattering probability. The coordinates $\mathrm{x}$ and $\mathrm{y}$ (Fig. 3) of the scattering event are sampled from an uniform distribution inside the converter area (both rectangular and circular area models were implemented), while the $z$ coordinate (distance of the scattering event from the exit surface) is sampled from an uniform distribution between 0 and $R^{\text {scint }}\left(E_{n}\right)$.

Below $10 \mathrm{MeV}$, only s-wave neutrons can interact with the nucleus, and the scattering angular distribution can be considered isotropic in the center-of-mass system. The scattering angle $\varphi$ is then sampled from a distribution between 0 and $2 \pi$.

In the laboratory system, $\cos ^{2} \theta$ is distributed uniformly from $\theta=0^{\circ}$ to $\theta=90^{\circ}$. Since the recoil-proton energy $E_{p}$ is given by $E_{n} \cdot \cos ^{2}(\theta)$, the uniform distribution with respect to $\cos ^{2}(\theta)$ 
corresponds to the uniform distribution with respect to $E_{p}$.

For the $\theta$ angle the probability density function is $\operatorname{pdf}\left(\cos ^{2} \theta\right)=1$. Since:

$p d f\left(\cos ^{2} \theta\right) d \cos ^{2} \theta=p d f(\theta) d \theta$

$p d f(\theta)=p d f\left(\cos ^{2} \theta\right) \frac{d \cos ^{2} \theta}{d \theta}=-2 \cdot \sin \theta \cdot \cos \theta=-\sin 2 \theta$

the cumulative distribution of the angle $\theta$ is:

$\operatorname{cdf}(\theta)=\int_{0}^{\theta}-\sin 2 \theta^{\prime} d \theta^{\prime}=\frac{1-\cos 2 \theta}{2}$

The stopping point of a recoil-proton and the deposited energies are then calculated from this six variables $\left(E_{n}, \mathrm{x}, \mathrm{y}, \mathrm{z}, \theta\right.$ and $\varphi$ ). Protons not reaching the $\Delta \mathrm{E}$ stage are discarded.

\subsection{Simulations results}

The spectrometer was simulated in a PCT configuration, with the polyvinyltoluene converter adjacent to the MST; the air volume was then neglected. Starting from a monoenergetic neutron source, the effect of the scattering reaction together with the energy loss of the recoil-protons inside the converter lead to a broad continuum response of the silicon detector, with energy varying from 0 to the neutron energy (Fig. 4). A $3.31 \mathrm{MeV}$ monoenergetic neutron source was simulated since experimental data are available for the PCT configuration (Agosteo and Pola, 2008).

In order to account for a device which could measure the recoilproton energy deposited in the converter (as it could with a scintillator converter), the energy lost by the recoil-proton in polyvinyltoluene was added to the one deposited in the silicon detector, thus calculating the total recoil-proton energy. This is representative of a device with a scintillator/converter strictly in contact with the MST.

As it shown in Fig. 5, a quasi-triangular distribution is obtained for the recoil-proton energy deposited in the three detectors constituting the spectrometer, with a maximum corresponding to the neutron source energy. This distribution is affected by the angular distribution of recoil-protons. Such a device, although providing an additional piece of information with respect to the classical PCT configuration, would require experimental data unfolding for reconstructing the neutron spectrum.

In order to obtain a narrow peak response (with a shape as close as possible to that of the monoenergetic neutron source), simulations were performed by separating the converter from the MST. The detector system was simulated in vacuum to avoid energy loss in air. Fig. 6 shows the distributions obtained at different distances between the converter and the MST.

The simulated distributions of Fig. 6 show an improvement in the response function. At a distance of $20 \mathrm{~mm}$, corresponding to an angle $\theta_{\max } \approx 7^{\circ}$ for the simulated system, the response shows an energy resolution of about $50 \mathrm{keV}$-FWHM. It should be mentioned that the MST resolution is $8 \mathrm{keV}-\mathrm{FWHM}$.

Fig. 7a-c show the simulated spectra of recoil-proton energy deposited in the converter, in the $\Delta \mathrm{E}$ stage and in the MST $(\Delta \mathrm{E}+\mathrm{E}$ stages).

Fig. 8 shows the scatter-plot of the energy deposited by recoilprotons for the $\mathrm{E}_{\Delta \mathrm{E}}-\mathrm{E}_{\mathrm{MST}}$ coupling (i.e $\Delta \mathrm{E}$ stage against $\Delta \mathrm{E}+\mathrm{E}$ stages). The linear part with unitary slope (left) is due to stoppers in the $\Delta \mathrm{E}$ stage; the inverse-proportional part (right) is due to crossers and reflects the proton stopping power behavior against proton energy.

The scatter plot for the $E_{\text {scint }}-E_{M S T}$ coupling is shown in Fig. 9. Apart from the contribution due to the titanium layer, all the energy is shared between the converter and the MST, so that the relation is linear with a negative unitary slope.

A response function so narrow in resolution (Fig. 6) allows to reconstruct the neutron spectrum from that of the recoil-protons by just applying the function of interaction probability in the

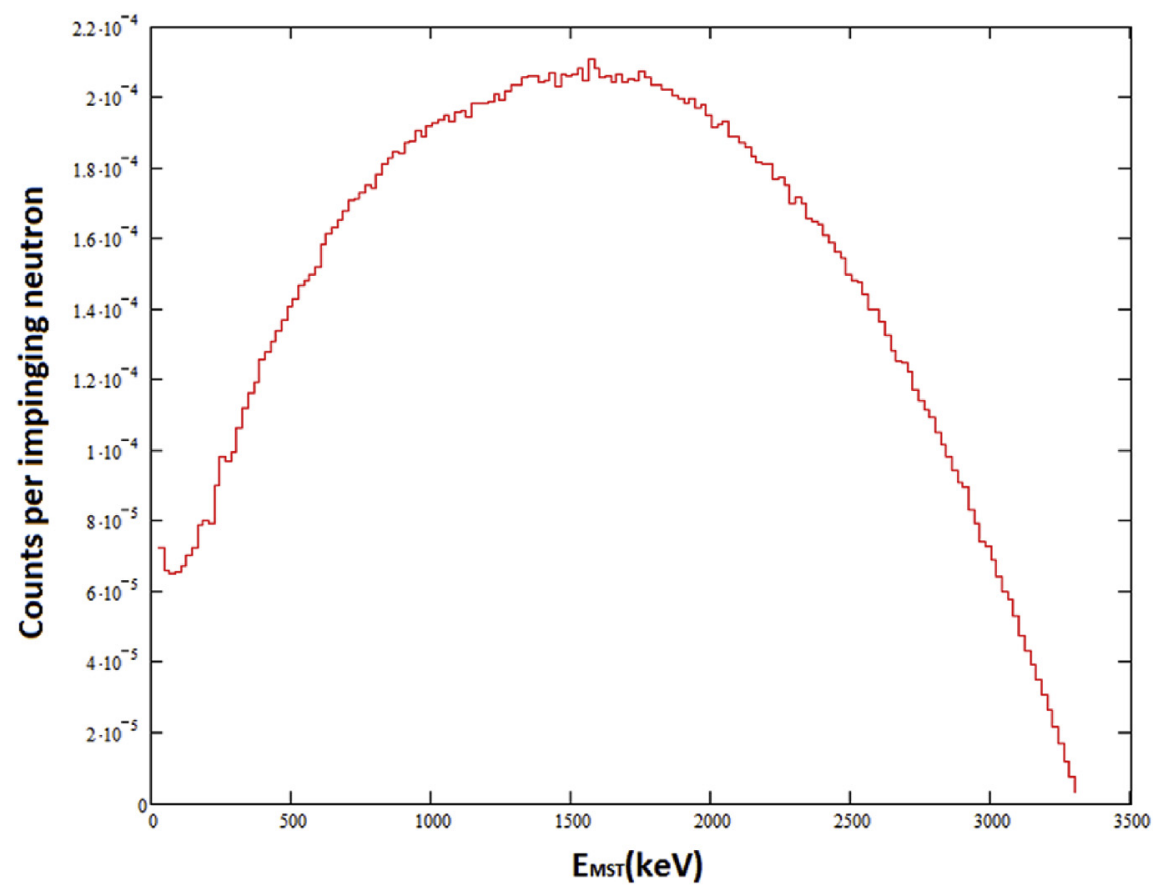

Fig. 4. Simulated response of the MST detector $(\Delta \mathrm{E}+\mathrm{E}$ stage $)$ to $3.31 \mathrm{MeV}$ neutrons. The scintillator/converter was simulated adjacent to the MST. 


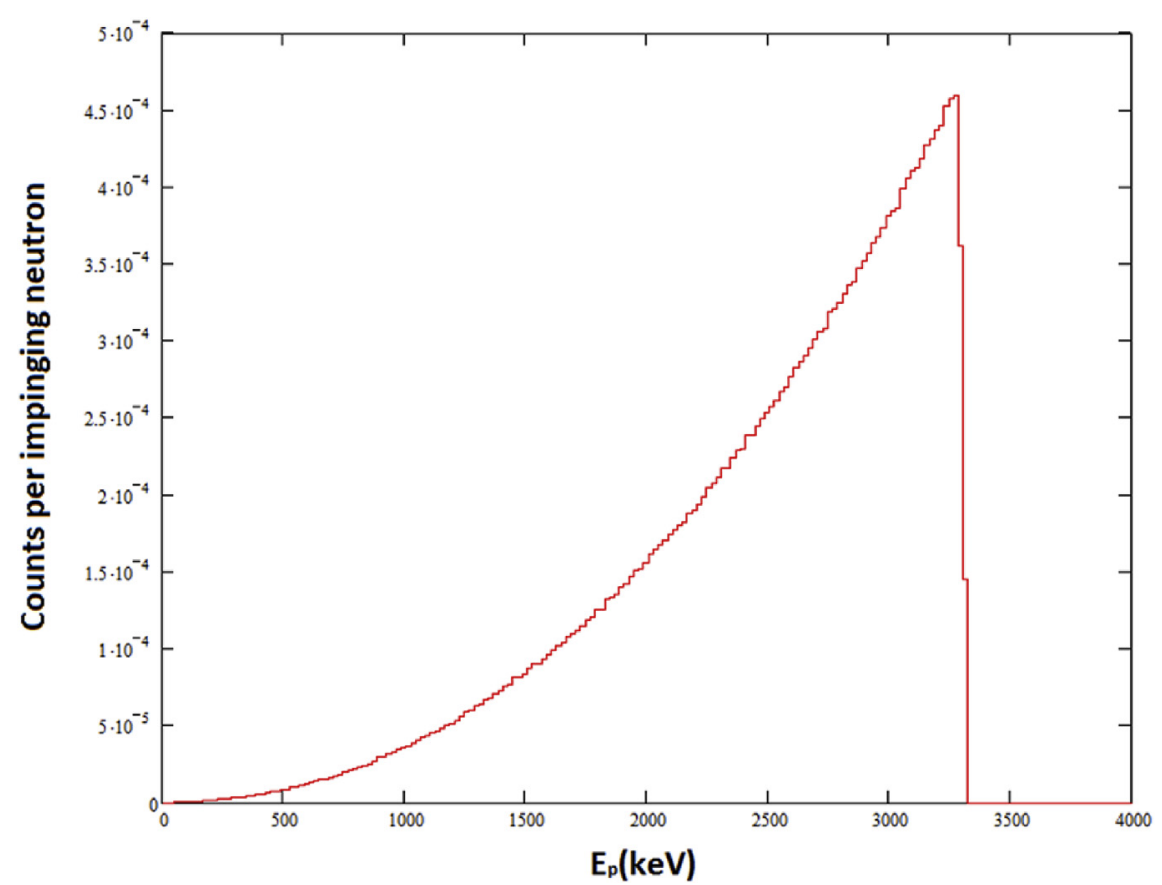

Fig. 5. Simulated response of the whole detection system (scintillator $+\Delta \mathrm{E}+\mathrm{E}$ stage) to $3.31 \mathrm{MeV}$ neutrons. The scintillator/converter was simulated adjacent to the MST.

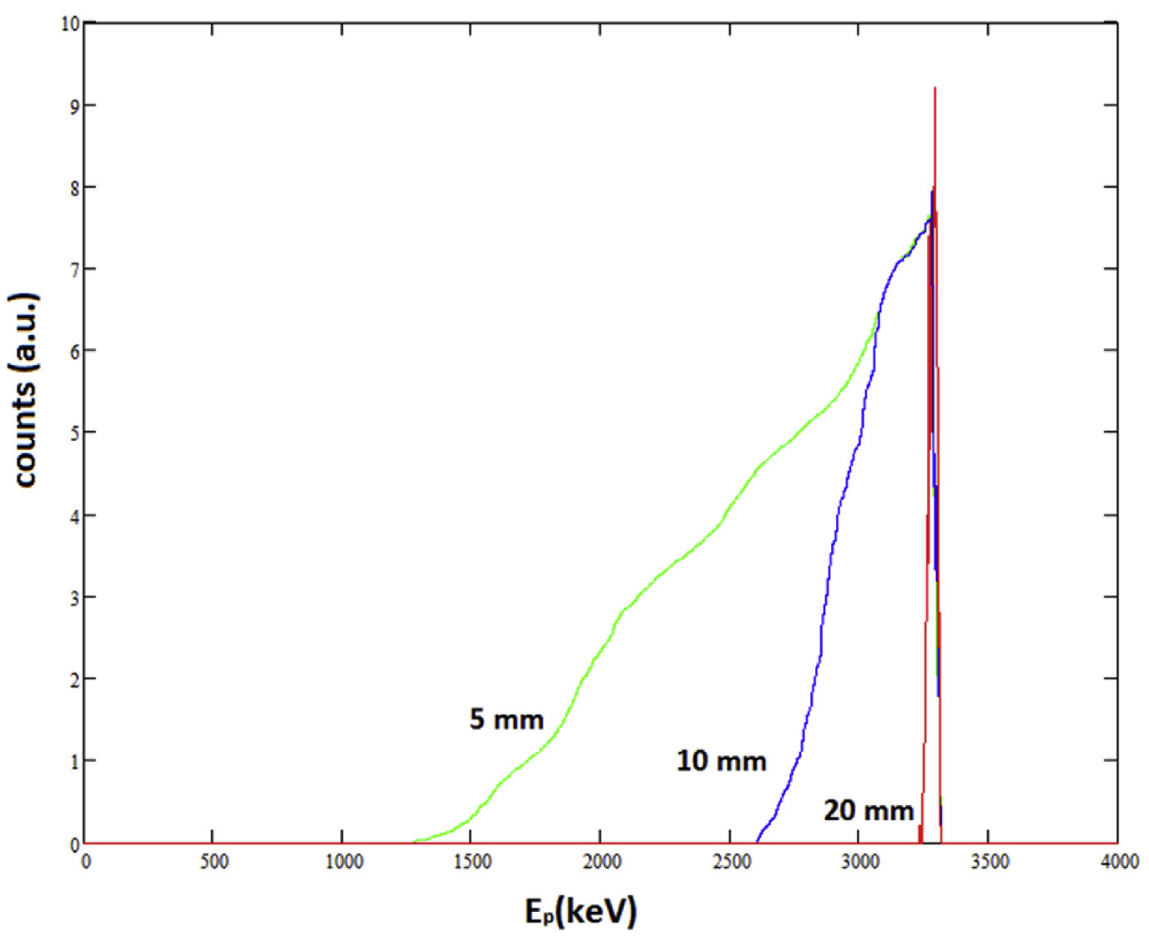

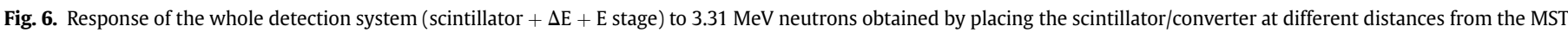

converter, without applying any unfolding procedure. This would solve the main problems that prevent the goals of a high-resolution and real-time spectrometer. It should be underlined that this high resolution goes to the detriment of the system sensitivity. Therefore, in general, a proper trade-off between the resolution and the system sensitivity must be identified on the basis of a preliminary evaluation of the spectral structure of the neutron field under investigation and the fluence rate expected.
In order to understand the feasibility of such a detecting system, the performance of different types of photomultipliers coupled with plastic scintillators was studied, as it is discussed in the next Section.

\section{Scintillator and photomultiplier}

The numerical study described in Sections 3 and 4 led to the 

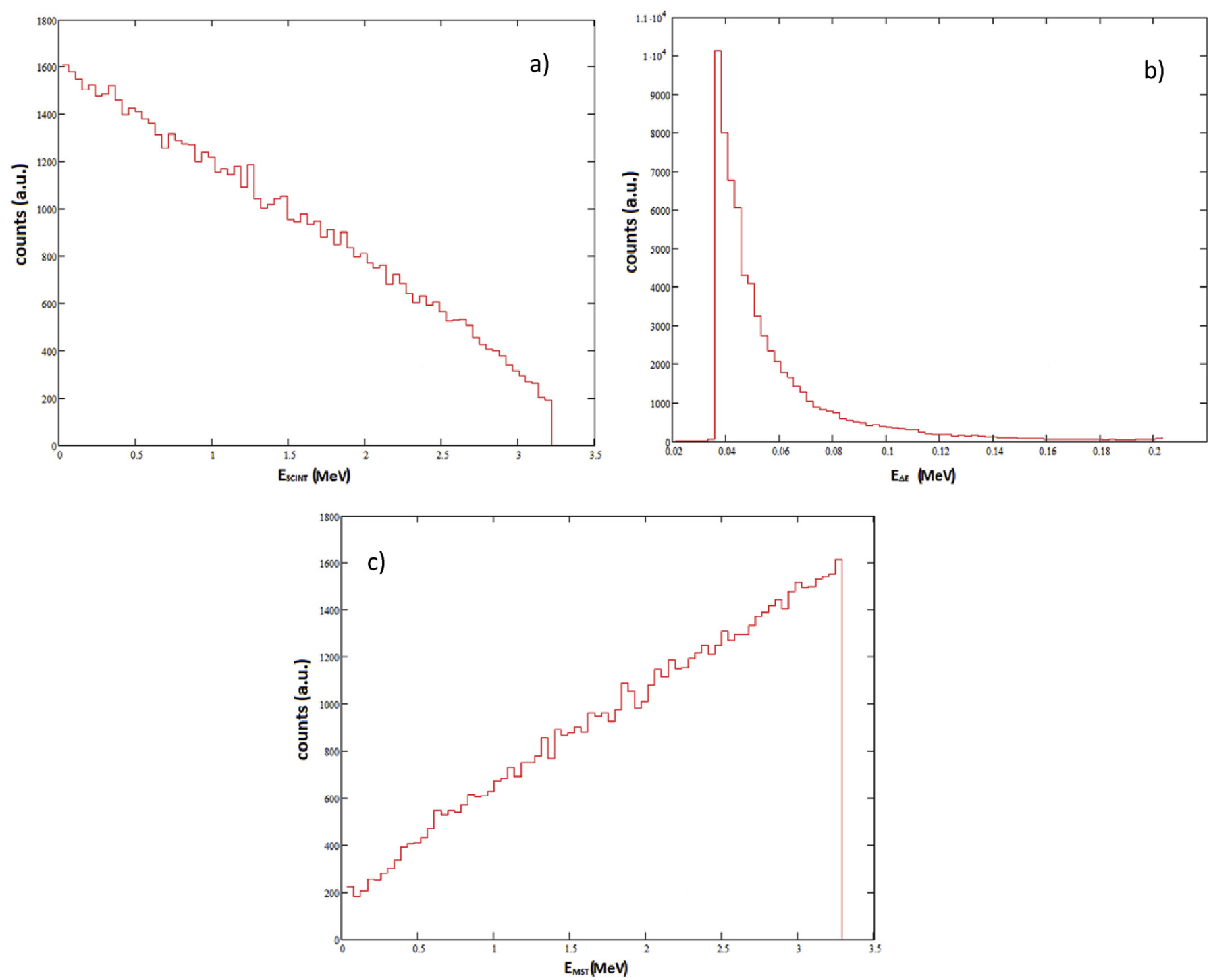

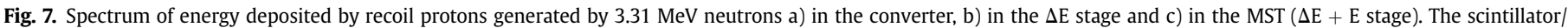
converter was simulated at $20 \mathrm{~mm}$ from the MST.

design of a detection system with narrow response functions to monoenergetic neutrons. This result gave confidence about the feasibility of a spectrometer capable of assessing neutron spectra without the use of unfolding procedures.

The main feature in the system layout is the adoption of an "active" converter which converts neutrons into recoil-protons via elastic scattering and measures the energy loss of the recoilprotons inside the converter itself at the same time. The latter information can be acquired with a photomultiplier stage coupled to a plastic scintillator. The selection of these devices should also account for the requirement of setting up a compact system.

The selected scintillator was of the type BC-404T ${ }^{\mathrm{TM}}$, fabricated by Saint-Gobain Crystals (France), which is a blue-emitting polyvinyltoluene based scintillator. This detector was selected by taking into account i) its high light output (68\% of Anthracene); ii) its fast response ( 0.7 ns of rise time, 1.8 ns of decay time), iii) its optimum matching between the maximum emission wavelength $(408 \mathrm{~nm})$ and the spectral response of the photomultiplier tube (PMT). It should be mentioned that this scintillator light output for protons is about one order of magnitude lower than that of electrons.

A $7.3 \times 9.5 \mathrm{~mm}^{2}$ portion was cut from a scintillator sheet $2 \mathrm{~mm}$ in thickness, in order to match the MST sensitive area. The lateral and the top faces (the latter is the one exposed to the neutron field) were covered with BC-620 ${ }^{\mathrm{TM}}$ paint (Saint-Gobain Crystals, France), a reflector based on titanium dioxide.

The scintillator-PMT interface was coupled by using the BC$630^{\mathrm{TM}}$ silicone optical grease (Saint-Gobain Crystals, France), which is a low evaporation compound with $1.06 \mathrm{~g} \mathrm{~cm}^{-3}$ in density and 1.465 in refractive index. These values are almost equal to the scintillator ones.

The selection of the photomultiplier tube for the active converter was based on the following requirements: i) small size to keep the whole system as compact as possible; ii) high sensitivity in the visible range to match the weak scintillator response to protons; iii) high speed response to manage high count rates (mainly due to photons associated to the neutron field).

A Hamamatsu photomultiplier tube H10720-110 Photonics, Japan) was selected. This device is a module containing a high-voltage power supply circuit and a photomultiplier tube R9880U-110 of its quantum efficiency falls in the $350-450 \mathrm{~nm}$ range, thus matching the $408 \mathrm{~nm}$ maximum emission value of the BC-404 ${ }^{\mathrm{TM}}$ 


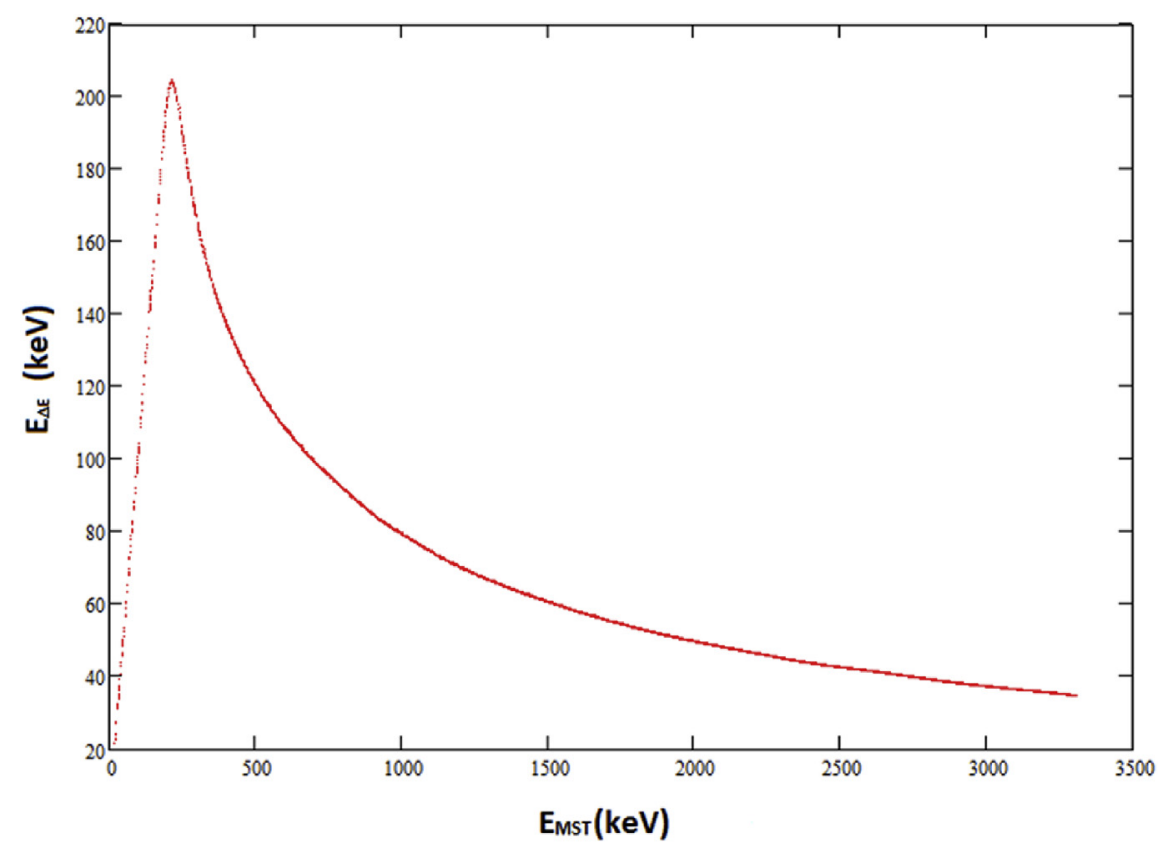

Fig. 8. Simulated $\Delta \mathrm{E}-\mathrm{E}$ scatter-plot (3.31 MeV neutrons). The scintillator/converter was simulated at $20 \mathrm{~mm}$ from the MST.

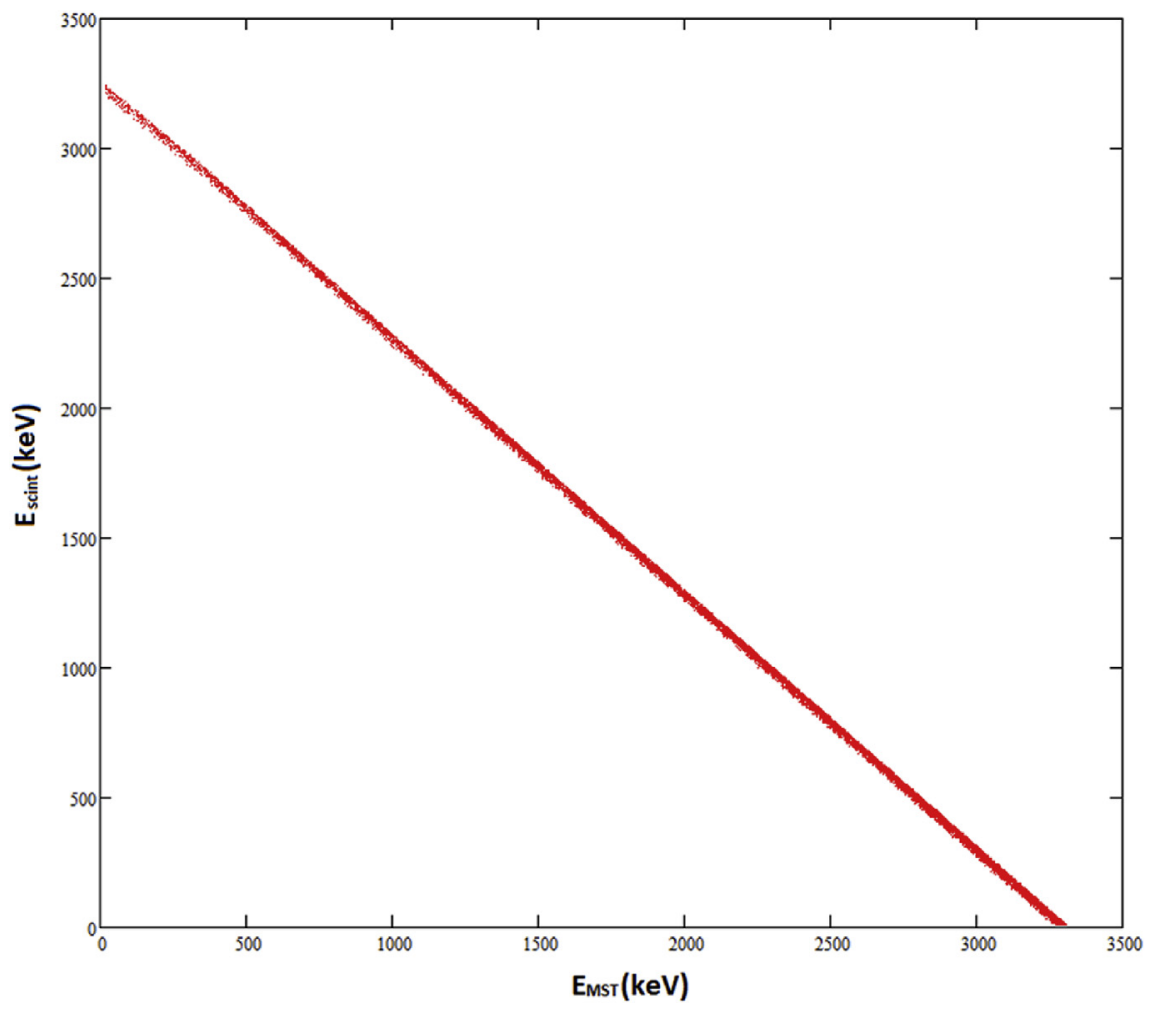

Fig. 9. Simulated $\mathrm{E}_{\mathrm{scint}}-\mathrm{E}_{\mathrm{MST}}$ scatter-plot for $3.31 \mathrm{MeV}$ neutrons. The scintillator/converter was simulated at $20 \mathrm{~mm}$ from the MST.

scintillator. This PMT package cannot operate in vacuum and therefore it had to be placed outside the vacuum chamber housing the detection system.

It should be mentioned that silicon photomultipliers (SiPM) (Ghioni et al., 2007; Zappa et al., 2007) were also considered and tested for converting the light from the scintillator. With respect to standard PMT, these semiconductor devices offer a higher quantum efficiency (defined as the average number of emitted photoelectrons per incident photon), fast response, lower power requirements and a small dimensions. A SiPM constituted by matrix of $60 \times 60$ cells, with a total active area of $10 \mathrm{~mm}^{2}$, coupled with a $\mathrm{BC}-404^{\mathrm{TM}}$ plastic scintillator was thoroughly characterized. The characterization of this device is beyond the scope of the present work. It should be stressed that the energy resolution for $662 \mathrm{keV}$ 
gamma rays from a ${ }^{137} \mathrm{Cs}$ source resulted to be better for the $\mathrm{BC}$ $404^{\mathrm{TM}}$ scintillator coupled with the H10720-110 ${ }^{\mathrm{TM}}$ PMT (about 5.2\%) than for the same scintillator coupled with the SiPM (about 29.4\%).

The BC-404 ${ }^{\mathrm{TM}}$ scintillator coupled to the SiPM was irradiated with $3.31 \mathrm{MeV}$ quasi-monoenergetic neutrons at INFN-Legnaro National Laboratory (LNL), Italy. The SiPM suffered from radiation damage after being irradiated with a fluence of $\sim 10^{9} \mathrm{~cm}^{-2}$, thus demonstrating to be unsuitable for operation in fast neutron fields, as confirmed by recent studies indicating deterioration with irradiation even after a few $10^{8} \mathrm{~cm}^{-2}$ (Angelone et al., 2010).

\subsection{Non-linearity of the scintillator/converter}

The selected configuration of the $\mathrm{BC}-404^{\mathrm{TM}}$ scintillator coupled with the H10720-110 ${ }^{\mathrm{TM}}$ PMT was also irradiated with $3.31 \mathrm{MeV}$ quasi-monoenergetic neutrons at the LNL. As expected, the scintillator/converter showed a non-linear behavior. Fig. 10 shows the scatter plot of the light generated by the scintillator against the energy $\mathrm{E}_{\mathrm{MST}}$ deposited by recoil-protons in the MST. The theoretical trend resulting from the simulations discussed in Section 4.1 is shown in the Fig. 10 (linear behavior).

This well-known non-linear behavior depends on the particle LET which affects the scintillation process by lowering the light conversion yield. The Birks' law (Birks, 1951, 1964; Knoll, 2010) describes the dependence of the light emitted per unit length $\mathrm{dL} / \mathrm{dx}$ on the stopping power $\mathrm{dE} / \mathrm{dx}$ of the interacting charged particle:

$\frac{d L}{d x} \propto \frac{\frac{d E}{d x}}{1+k_{B} \cdot \frac{d E}{d x}}$ where $\mathrm{k}_{\mathrm{B}}$ is the Birks' constant, which depends on the scintillating material $\left(0.088 \mathrm{~mm} \mathrm{keV}^{-1}\right.$ for polyvinyltoluene-based scintillators, Torrisi, 2000).

The integration of equation (18) gives:

$L=\int_{0}^{E_{p}} \frac{1}{1+k_{B} \cdot\left(\frac{d E}{d x}\right)^{p o l y}(E)} d E$

where $L$ is the generated light, $E_{p}$ is the proton energy, $(d E / d x)^{\text {poly }}(E)$ is the proton stopping power in polyvinyltoluene as a function of the proton energy.

This model represents the light generated by a proton which stops in the converter. Fig. 11 shows the curves obtained with

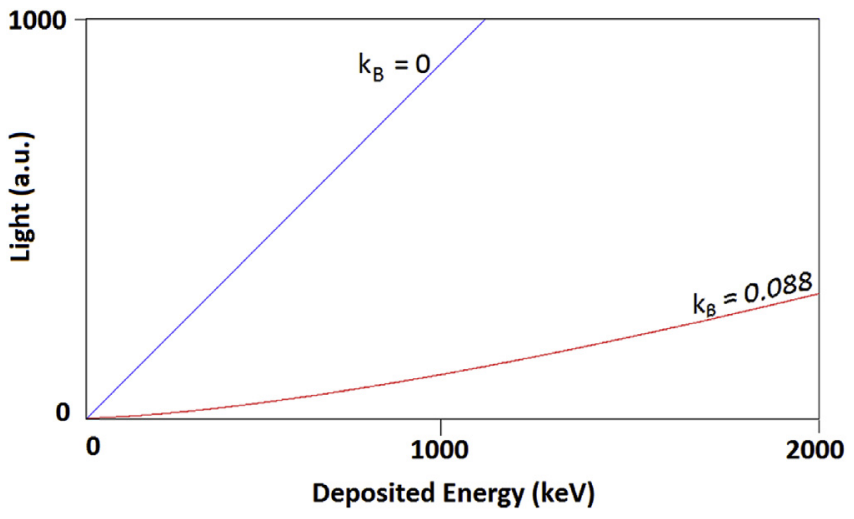

Fig. 11. Expected relation between the light generated by a proton in the scintillator at different deposited energies for $\mathrm{k}_{\mathrm{B}}=0$ and for $\mathrm{k}_{\mathrm{B}}=0.088 \mathrm{~mm} \mathrm{keV}^{-1}$.

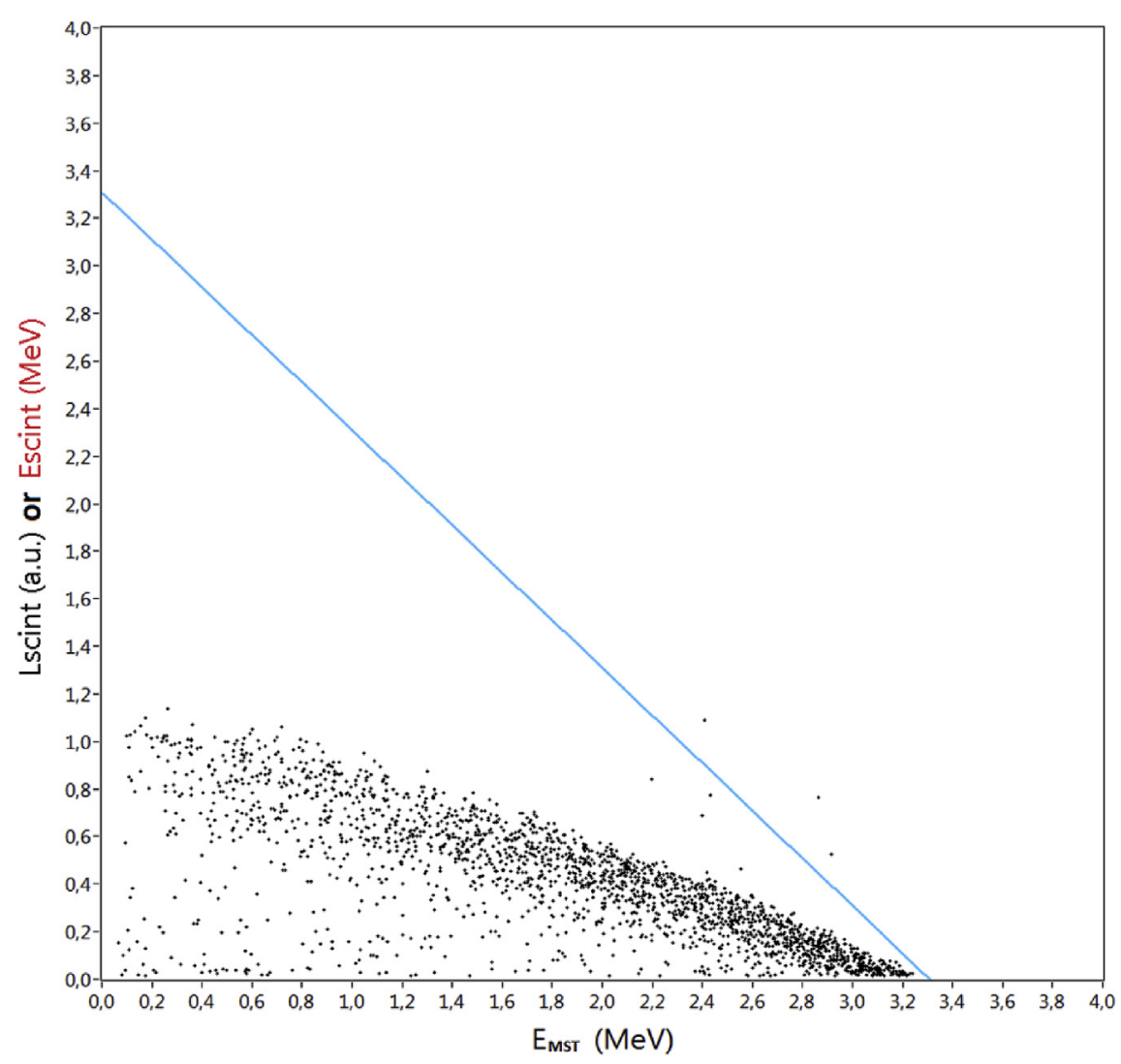

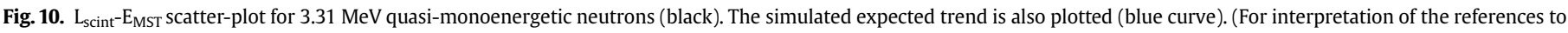
color in this figure legend, the reader is referred to the web version of this article.) 
equation (19), by setting $\mathrm{k}_{\mathrm{B}}=0$ and $\mathrm{k}_{\mathrm{B}}=0.088 \mathrm{~mm} \mathrm{keV}^{-1}$.

\subsection{Correction procedure}

The non-linearity of the scintillator response was corrected through an analytical procedure. This procedure derives directly the total recoil-proton energy $E_{p}$ from both the measured quantities: the light emitted in the scintillator $L_{S C I N T}$ and the energy deposited in silicon $E_{M S T}$ (Fig. 12).

Through equation (19) with $\mathrm{k}_{\mathrm{B}}=0.088 \mathrm{~mm} \mathrm{keV}^{-1}$, the algorithm calculates the equivalent quantity of light $L_{E Q}$ corresponding to the residual recoil-proton energy $E_{M S T}$ measured by the telescope. In other words, $L_{E Q}$ is the light which should be generated in the scintillator by a proton of energy $E_{M S T}$. The quantity $L_{E Q}$ is then added to $L_{S C I N T}$ (the light actually measured) to estimate the total light $L_{P}$ generated by a recoil-proton of energy $E_{p}$. Therefore, $L_{p}=L_{S C I N T}+L_{E Q}$ corresponds to the total light which should be generated if the recoil-proton stopped completely inside the scintillator.

Finally, the total recoil proton energy $E_{p}$ is derived from $L_{p}$ by applying the inverse function of equation (19), calculated numerically.

The energy $E_{S C I N T}$ deposited in the converter can be calculated by subtracting $E_{M S T}$ from $E_{p}$.

The results obtained by applying the correction algorithm to the data measured at the LNL are shown Fig. 13 (red points). The Fig. 13 shows the analytical curves obtained by accounting for a linear $\left(\mathrm{k}_{\mathrm{B}}=0\right)$ and a non-linear $\left(\mathrm{k}_{\mathrm{B}}=0.088 \mathrm{~mm} \mathrm{keV}^{-1}\right)$ light response of the scintillator/converter. The experimental data spread is mainly due to energy straggling and fluctuations of electron multiplication in the PMT, which were neglected in the simulations.

\section{Spectrometer layout}

As discussed in the previous Sections, the active converter spectrometer (ACSpect) consists of a combination of two stages: the active converter based on a plastic scintillator and a Monolithic Silicon Telescope which acts as a recoil-proton residual energy stage. The MST is $1 \times 1 \mathrm{~mm}^{2}$ in sensitive area.

The converter and the MST were housed in a small aluminum vacuum chamber (dimensions $52 \times 38 \times 21 \mathrm{~mm}^{3}$ ). The chamber was equipped with a vacuum connector and an aluminum pipe $4 \mathrm{~mm}$ in diameter and $2 \mathrm{~cm}$ in length for collimating recoil-protons from the converter to the MST. The collimator was provided at one end with an O-ring. The scintillator/converter was finally coupled to the O-ring. Thus, collimated recoil-protons enter directly the vacuum region, cross the collimation channel and impinge on the

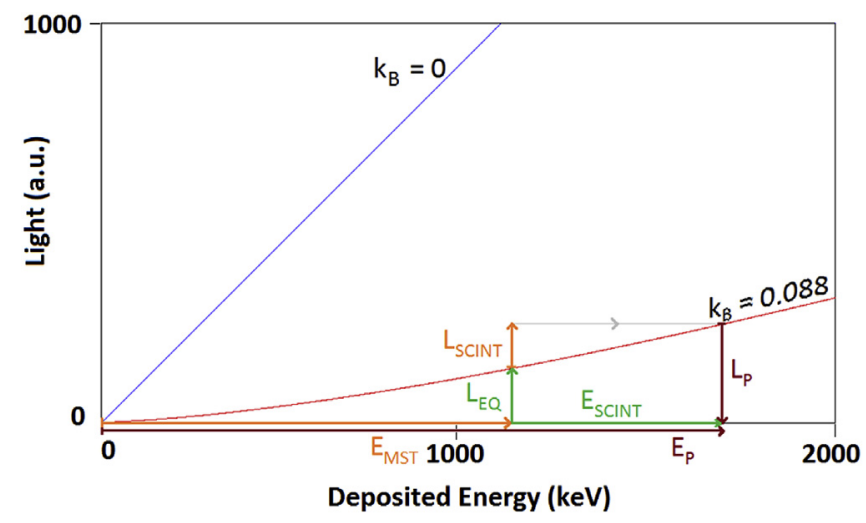

Fig. 12. Scheme of the procedure for reconstructing the total recoil-proton energy.
MST. This configuration also grants the alignment between the converter and the MST. The actual distance between the converter and the MST is $21 \mathrm{~mm}$. It should be observed that only recoilprotons in the scattering angular range $\theta=0^{\circ}-7.35^{\circ}$ $\left(\theta_{\max }=\tan ^{-1}((2+\sqrt{2} / 2) / 21) \cong 7.35^{\circ}\right)$ can interact with the MST after passing through the collimator. Therefore, from equation (1), the uncertainty on the recoil-proton energy due to physical collimation is $\pm 1.64 \%$ (i.e., $1-\cos ^{2} 7.35^{\circ}$ ).

The front-end electronics of the MST consists of two custom charge sensitive preamplifiers, designed and assembled at the Nuclear Electronics Laboratories of the Department of Energy of the Politecnico di Milano. The preamplifiers were connected to the MST in the so called $\Delta \mathrm{E}-\mathrm{E}_{\mathrm{MST}}$ configuration, i.e. the charge is collected from the $\Delta \mathrm{E}$ stage (to a preamplifier with an injection capacitance of $2.7 \mathrm{pF}$ ) and from the $\mathrm{p}^{+}$common layer (preamplifier capacitance $\mathrm{C}_{\mathrm{inj}}=10.34 \mathrm{pF}$ ) in order to acquire directly the total energy deposited inside the MST.

All the components were housed in a $122 \times 172 \times 54 \mathrm{~mm}^{3}$ aluminum box, which also couples the MST-converter vacuum case to the PMT. Fig. 14 shows the final ACSpect assembly.

During the measurements, the MST stages were connected to separate electronic chains. In particular, the common $\mathrm{p}^{+}$layer and the E stage were biased at $-6 \mathrm{~V}$ and $+150 \mathrm{~V}$, respectively. The $\Delta \mathrm{E}$ stage and the $\mathrm{p}^{+}$layer were connected to two standard Ortec $671^{\mathrm{TM}}$ amplifiers. The shaping times were set at $3 \mu$ s and $2 \mu$ s for the $\Delta \mathrm{E}$ stage and the $\mathrm{p}^{+}$layer, respectively, in order to maximize the signal to noise ratio.

The PMT was powered with a $+5 \mathrm{~V}$ source, and the output signals were amplified with a Silena $7611^{\mathrm{TM}}$ standard amplifier, with $250 \mathrm{~ns}$ in shaping time.

The output signals of the three chains were acquired by a commercial fast digitizer PicoScope 4424 ${ }^{\mathrm{TM}}$ (Pico Technology LtD., United Kingdom), controlled by a software developed at the $\mathrm{Nu}$ clear Measurement Laboratory of the Politecnico di Milano Energy Department. The device resolution is 12 bit and it was set to acquire signals from the three input channels at a sampling frequency of $20 \mathrm{MS} \mathrm{s}^{-1}$, by using the $\Delta \mathrm{E}$ signals as trigger events.

A scheme of the whole ACSpect chain is shown in Fig. 15.

\section{Calibration}

The $\Delta \mathrm{E}$ and $\mathrm{E}_{\mathrm{MST}}$ chains were calibrated through an electronic procedure. The deposited energy $E$ and the charge $Q$ generated in silicon are related through:

$E=\frac{Q \cdot \varepsilon_{S i}}{e}$

where $e$ is the electron charge $\left(1.602 \cdot 10^{-19} \mathrm{C}\right)$ and $\varepsilon_{S i}$ is the mean energy required to create an electron-hole pair in silicon $(3.62 \mathrm{eV})$.

If the value of a test capacitance $C_{i n j}$ is known accurately, the charge generated by a signal test of amplitude $V_{\text {test }}$ could be evaluated as:

$Q_{\text {test }}=V_{\text {test }} \cdot C_{\text {inj }}$

and therefore, from equations (20) and (21), the equivalent energy $E_{\text {test }}$ can be estimated as:

$E_{\text {test }}=\frac{V_{\text {test }} \cdot C_{i n j} \cdot \varepsilon_{S i}}{e}$

The chain can thus be calibrated in energy with test pulses of different amplitudes.

The signals from the PMT are not linear against deposited energy, as it was discussed in Section 5.1. Equation (19) relates the 


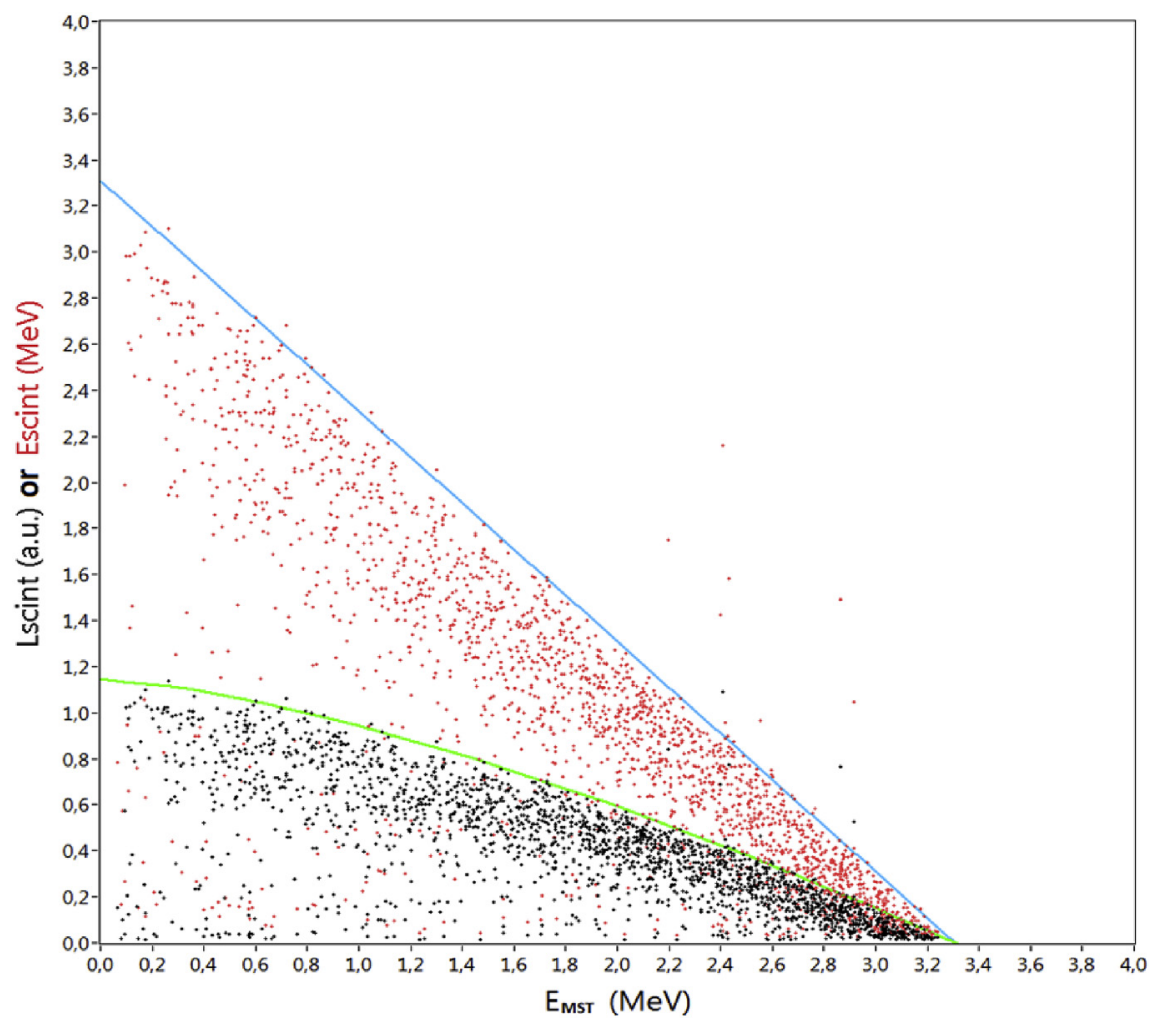

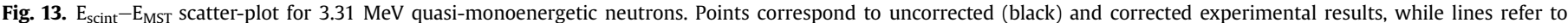
simulated non-linear and linear trends.

energy to the light emitted by the scintillator. The simulated nonlinear curve of the scatter-plot shown in Fig. 13 (green line (in the web version)) is used for calibrating the acquired signals from the scintillator (Fig. 13, black dots), thus providing corrected data which are calibrated against energy.

\section{Data processing}

A LabVIEW ${ }^{\mathrm{TM}}$ software was developed for performing a realtime spectrometry capable of processing: data sampling and acquisition, triple timing coincidence, particle discrimination and non-linear light generation correction.

As it was mentioned in Section 6, the output signals of the ACSpect were acquired with a PicoScope $4424^{\mathrm{TM}}$. The oscilloscope control and data sampling procedures have been developed with libraries provided by Pico Technology, United Kingdom.

The processing software manages in real-time:

- signal peak search, basing on a quadratic fit;

- energy calibration (energy for $\Delta \mathrm{E}$ and $\mathrm{E}_{\mathrm{MST}}$ stages and arbitrary light units for the PMT);

- triple coincidence among the three detector/stages and discrimination of the proton-related events;

- non-linear light generation correction procedure;

- calculation and display of spectra related to accepted and rejected events from the three stages, corrected events for the converter, total energy events for protons, neutron spectrum and $2 \mathrm{D}$ scatter plots related to $\Delta \mathrm{E}-\mathrm{E}_{\mathrm{MST}}$ distribution, $\mathrm{E}_{\mathrm{MST}}-\mathrm{E}_{\mathrm{scint}}$ distribution.

The software is capable of performing post-processing for time coincidence analysis and 3D scatter plot generation.
The software performance was tested while characterizing the ACSpect with $3.31 \mathrm{MeV}$ quasi-monoenergetic neutrons at the LNL. Fig. 16 shows the data processed by the time coincidence analysis routine, which calculates the distribution of events in terms of time of arrival with respect to the trigger reference. The plot in Fig. 16a shows the time distribution of events from the PMT against those from the $\Delta \mathrm{E}$ stage. Part of these events resulted to be concentrated in a well defined region, while the remainder was distributed sparsely over a wide time interval. The former population of events is due to recoil-protons generated inside the scintillator and interacting with the MST with a clear time correlation (an average delay of about $8 \mu \mathrm{s}$ ). The latter can be associated to uncorrelated events from the PMT and MST (random coincidence) generated by the intense $\gamma$-ray background. The distribution of proton-related events can be isolated by performing a time-energy shaped coincidence (gray lines) as it is shown in Fig. 16b. This "cleaned" distribution still includes undesired events, occurring mainly at low $\Delta \mathrm{E}$ energies.

The $\Delta \mathrm{E}-\mathrm{E}_{\mathrm{MST}}$ events can be selected through a time coincidence procedure which accepts the $\mathrm{E}_{\mathrm{MST}}$ events falling in a fixed time interval with respect to the $\Delta \mathrm{E}$ events; the proton events are discriminated through the $\Delta \mathrm{E}-\mathrm{E}_{\mathrm{MST}}$ scatter-plot (Fig. 17) where the recoil-proton distribution (black dots) can be easily discriminated (black lines) from other events (gray dots).

The processing software allows to derive the distribution of events due to recoil-protons only in a very effective way, through the discrimination procedures described above $\left(\Delta \mathrm{E}-\mathrm{E}_{\mathrm{MST}}\right.$ scatter plot and $\Delta$ E-PMT time coincidence), as it is shown in Fig. 18 (before (a) and after (b) discrimination).

A post-processing analysis of the signals acquired during the measurement was also developed. This algorithm calculates the time-energy distributions in the $\mathrm{E}_{\mathrm{MST}}$ stage and in the converter 


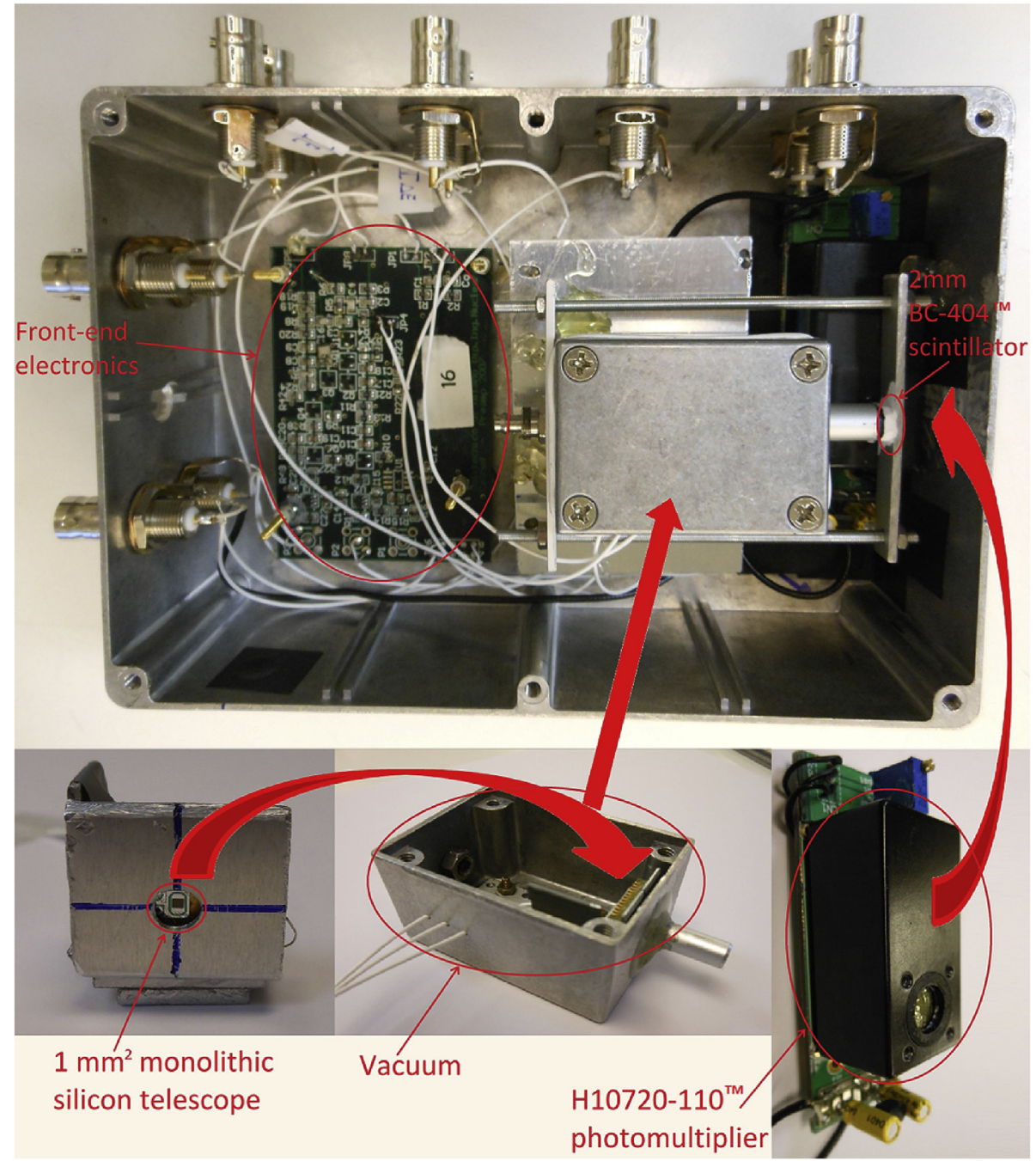

Fig. 14. A picture of the ACSpect system together with zooms of main parts.

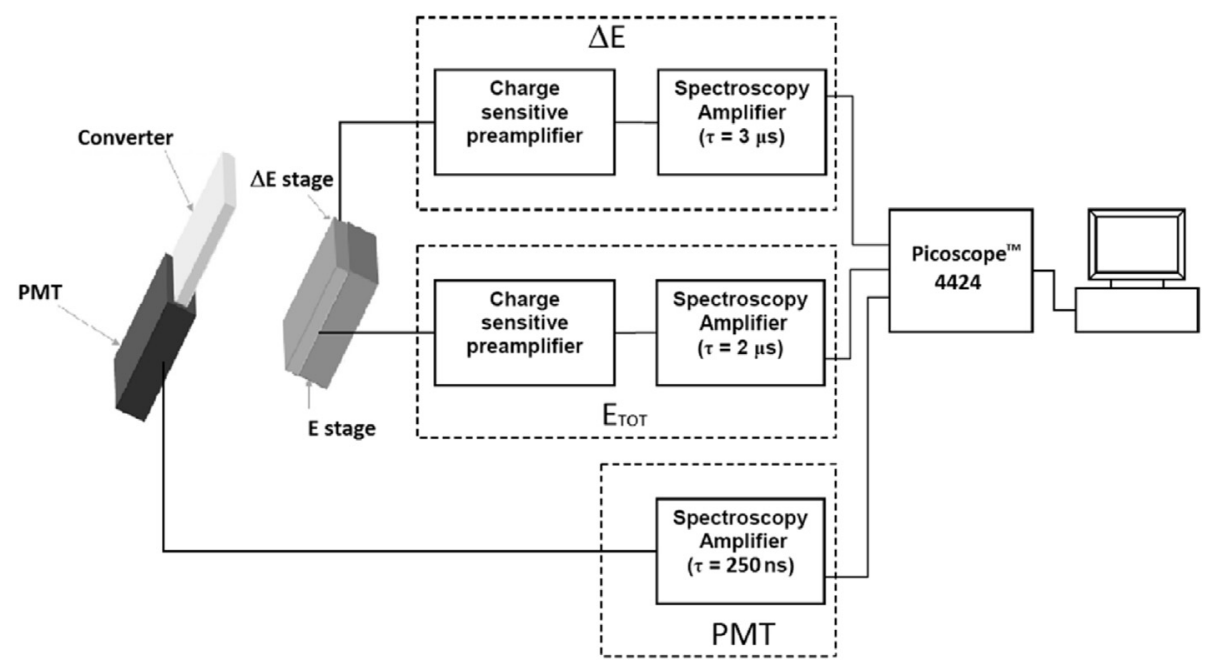

Fig. 15. Scheme of the ACSpect electronic chain.

occurring in a selected time interval with respect of the $\Delta \mathrm{E}$ event time occurrence.
The correction procedure for non-linearity in light generation (Section 5.2) was implemented with the LabVIEWтм code by using 

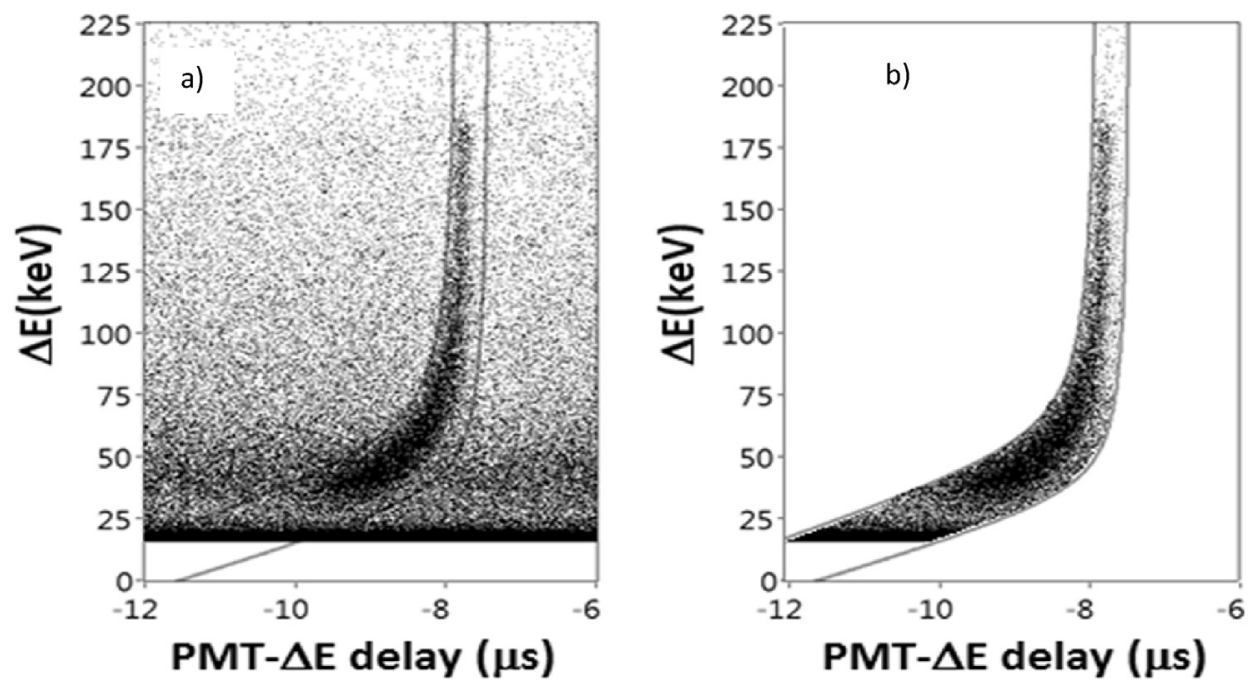

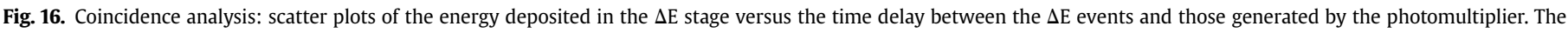

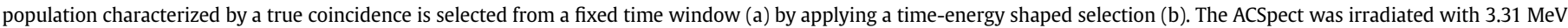
neutrons.

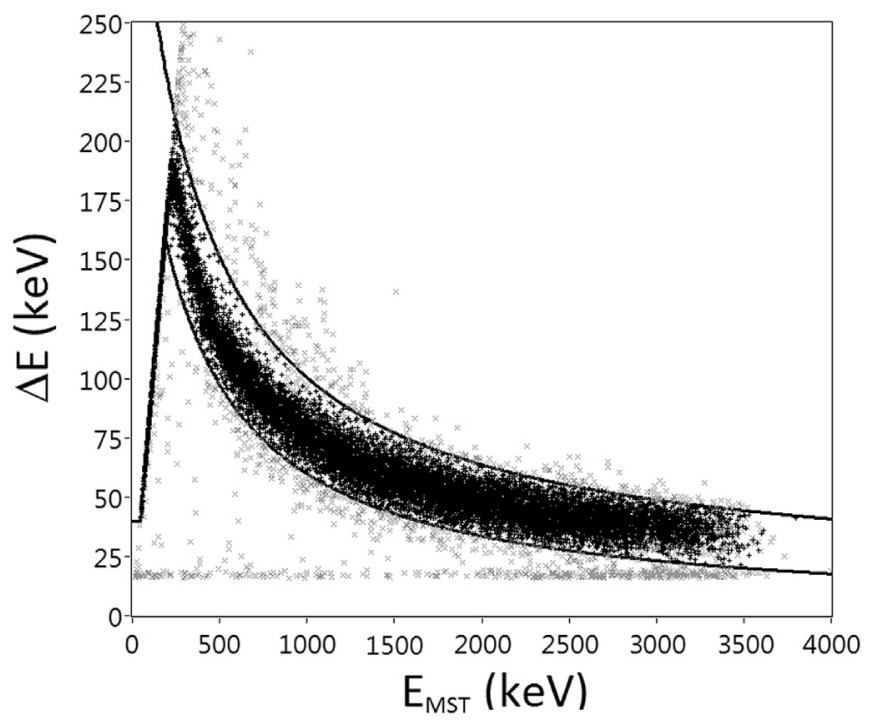

Fig. 17. Experimental $\Delta \mathrm{E}-\mathrm{E}_{\mathrm{MST}}$ scatter plot obtained by irradiating the system with 3.31 MeV neutron irradiation before (gray points) and after (black points) the coincidence procedure shown in Fig. 16. The black curves delimit the physical region where recoil protons are distributed.

the proton stopping power tables from ICRU 49 report (ICRU, 1993).

Finally, the neutron spectrum is obtained by dividing the recoilproton total energy distribution by the probability $\Pi\left(E_{n}\right)$ of generating a recoil-proton in polyvinyltoluene (equation (14)).

The data processing software gives: the spectra related to accepted and rejected events, energy distributions of accepted events, final recoil-proton spectrum and neutron spectrum. It also generates $2 \mathrm{D}$ scatter plots of $\Delta \mathrm{E}-\mathrm{E}_{\mathrm{MST}}$ distributions, $\mathrm{E}_{\mathrm{MST}}-\mathrm{E}_{\text {scint }}$ distributions and, in post-processing, also 3D scatter plots for the energy and time distribution analysis (Fig. 19).

\section{Spectrometer characterization}

The ACSpect was characterized with quasi-monoenergetic and continuous neutron fields at the $\mathrm{CN}-\mathrm{Van}$ De Graaff accelerator of the LNL. It should be remembered that no unfolding procedure was applied to reconstruct the neutron spectra.

\subsection{Quasi-monoenergetic neutrons}

Quasi-monoenergetic neutrons were generated from a thin LiF target $\left(700 \mu \mathrm{g} \mathrm{cm}{ }^{-2}\right.$ in thickness) via the ${ }^{7} \mathrm{Li}(\mathrm{p}, \mathrm{n})^{7} \mathrm{Be}$ reaction (threshold energy $1.88 \mathrm{MeV})$. The ${ }^{7} \mathrm{Li}(\mathrm{p}, \mathrm{n})^{7} \mathrm{Be}^{*}$ reaction channel also contributes to neutron production from the $0.430 \mathrm{MeV}$ excited level of ${ }^{7} \mathrm{Be}$, with a threshold energy equal to $2.13 \mathrm{MeV}$.

The spectrometer was placed at $0^{\circ}$ with respect to the proton beam direction. The distance between the target and the converter was $17 \mathrm{~mm}$. This led to a detection efficiency of the order of $10^{-6}$ counts per unit neutron fluence.

The charge of the accelerated proton beam was measured with a Faraday cup connected to a charge integrator.

The energy of the quasi-monoenergetic fields was $0.636,1.272$, 2.702, 3.309 and $3.914 \mathrm{MeV}$.

Fig. 20 shows the experimental scatter plot of the light $L_{\text {Scint }}$ emitted in the scintillator against the energy $\mathrm{E}_{\mathrm{MST}}$ deposited in the MST for all considered quasi-monoenergetic neutron fields. The theoretical curves from the analytical model are also plotted in the same Fig. and are in a satisfactory agreement with the experimental data.

The distributions of energy deposited in the three different stages of the ACSpect, i.e. scintillator/converter, $\Delta \mathrm{E}$ stage and $\mathrm{E}$ stage, are shown in Fig. 21(a-c) together with the distributions calculated with the analytical model. Again the agreement between the experimental and the theoretical data is satisfactory.

The neutron yield of $2.3 \mathrm{MeV}$ neutrons is shown in Fig. 22. The measured energy distribution was fitted with two Gaussian curves. The peak referring to the ${ }^{7} \mathrm{Be}^{*}$ reaction channel was fitted by assuming the same variance obtained for the main peak and by considering the expected energy difference $(0.48 \mathrm{MeV})$ and fluence ratio (0.055). With respect to the fitting curve, the measured spectrum shows a low energy tail. This may be due to the momentum spread of the accelerated protons, the energy-loss of the accelerated protons in the LiF target, energy straggling of recoilprotons and the roughness of the active converter surface.

Fig. 23 shows the neutron yield of the measured quasimonoenergetic neutron fields provided by data processing in 

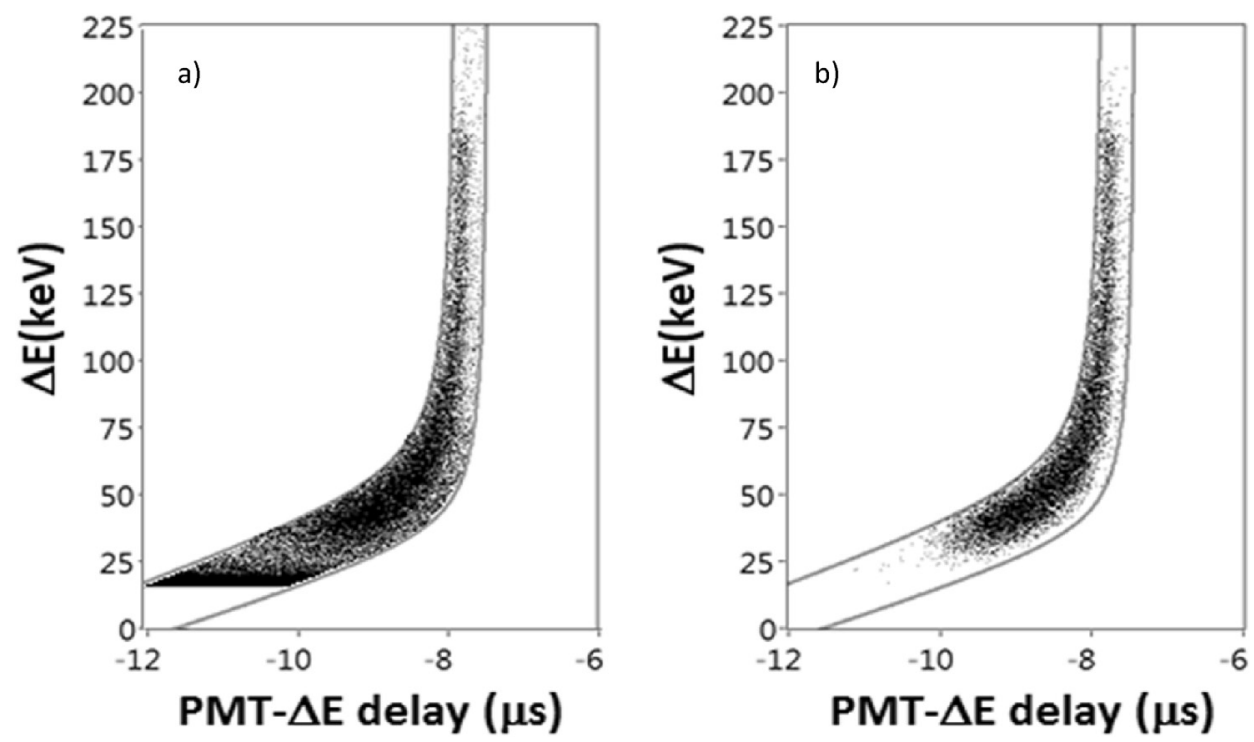

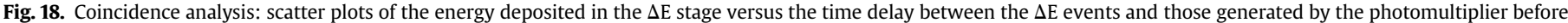
(a) and after (b) the event selection based on the $\Delta \mathrm{E}-\mathrm{E}_{\mathrm{MST}}$ Scatter plot (Fig. 17). The ACSpect was irradiated with $3.31 \mathrm{MeV}$ neutrons.

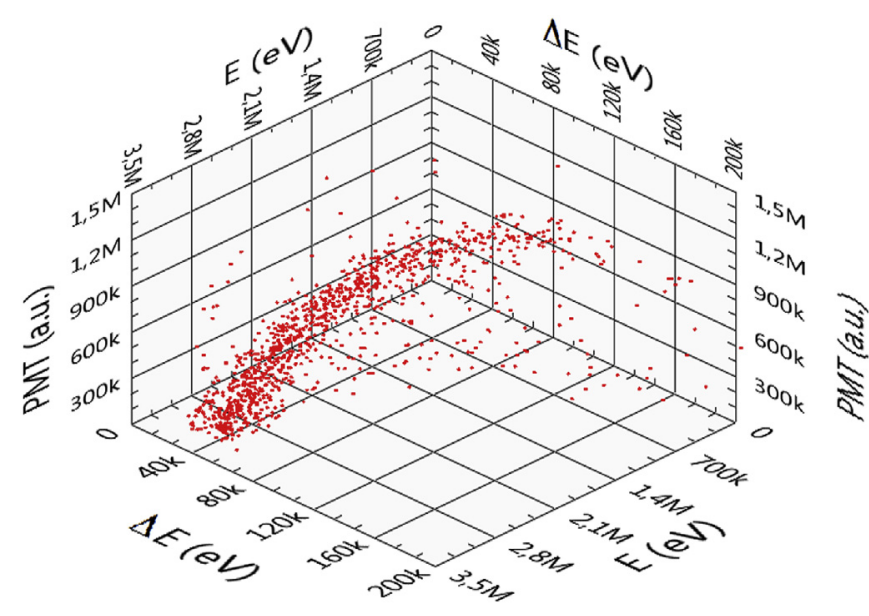

Fig. 19. 3D scatter plot of the information collected by the system (PMT, $\Delta E$ and E), event-by-event, (neutron energy $3.31 \mathrm{MeV}$ ). real-time.

The energy resolution of the spectrometer resulted to be about $235 \mathrm{keV}-\mathrm{FWHM}$ at $3.31 \mathrm{MeV}$, which corresponds to about 7\%. It should be noted that this value also includes the energy resolution of the quasi-monoenergetic field, which is not known at the LNL.

The results obtained with the ACSpect can be compared with those by Tomita et al., 2010. The spectrometer proposed by that group consists of a thin plastic scintillator which acts as a neutron converter, a silicon surface barrier detector (Si-SBD) used for recoil proton detection and a second thick plastic scintillator for detecting the scattered neutrons. The radiator works as a $\Delta \mathrm{E}$ detector and as fast timing detector for the time of flight measurements. Part of the system is set in vacuum to avoid recoil-proton energy loss. The energy of the recoil proton is given by the sum of the energy deposited in the thin plastic scintillator and in the Si-SBD, which is like a recoil proton telescope. In parallel, the energy of the scattered neutrons is measured with the ToF technique.

The device by Tomita et al., 2010 was used to measure $2.5 \mathrm{MeV}$ neutrons from the $\mathrm{D}-\mathrm{D}$ reaction. The measured spectrum was

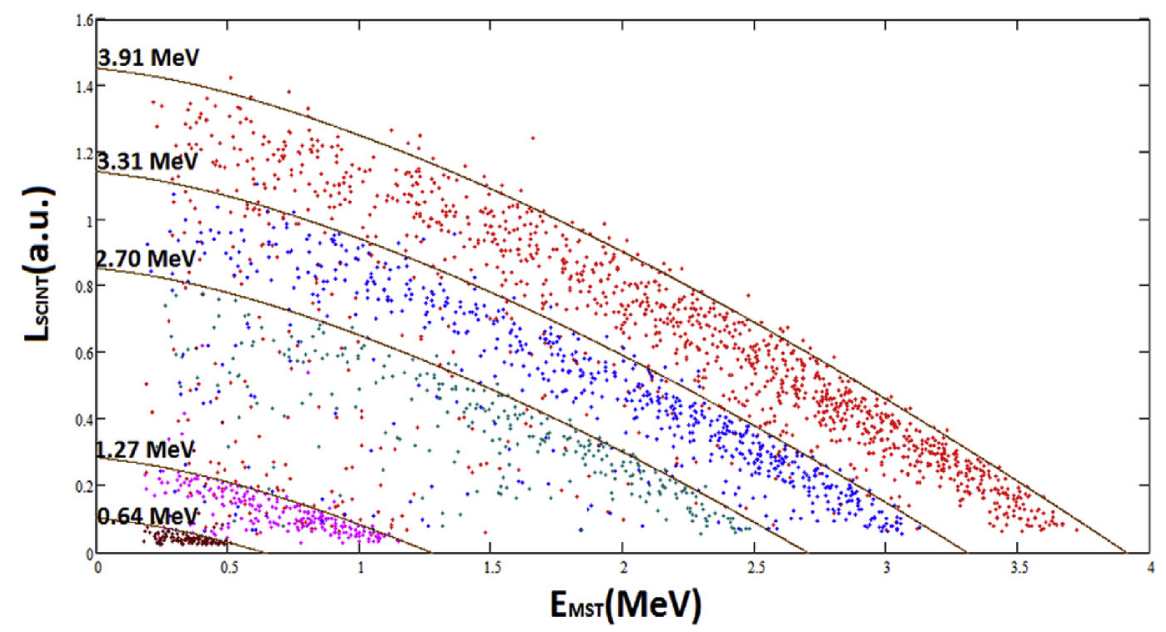

Fig. 20. $\mathrm{L}_{\mathrm{SCINT}}-\mathrm{E}_{\mathrm{TOT}}$ scatter plot for quasi-monoenergetic neutrons at different energies. The analytical curves corresponding to recoil protons emitted at $0^{\circ}$ are also plotted. 

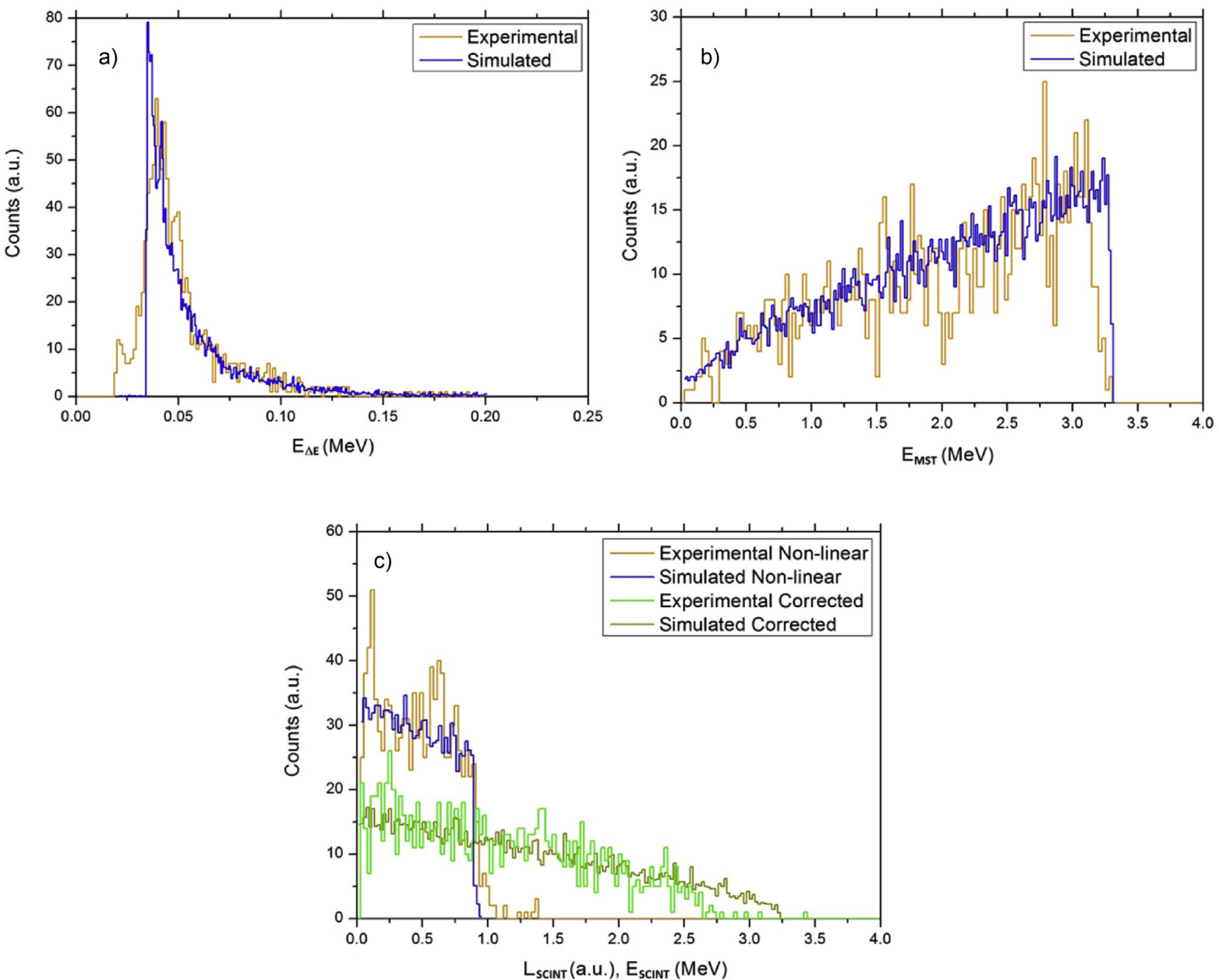

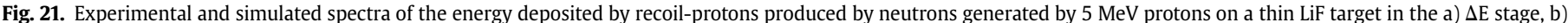
MST ( $\Delta E+E$ stage) and c) scintillator (before and after non-linearity correction).

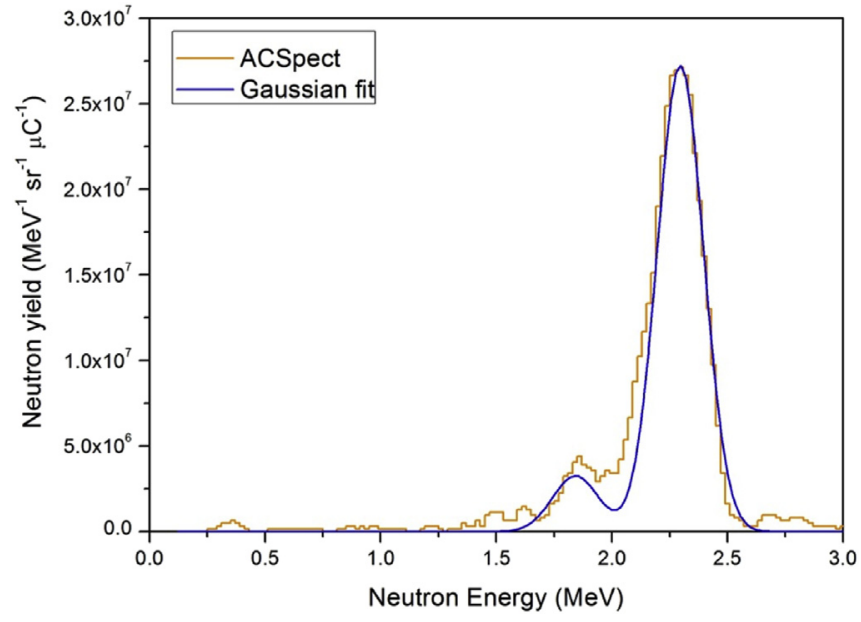

Fig. 22. Energy distribution of the yield of quasi-monoenergetic neutrons generated at $0^{\circ}$ by $4.0 \mathrm{MeV}$ protons on a thin LiF target.

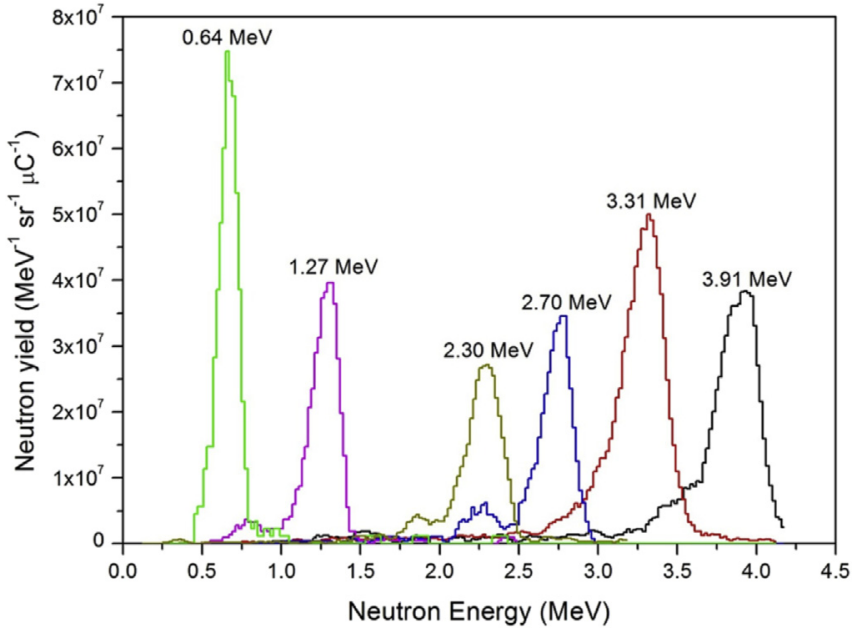

Fig. 23. Energy distribution of the yield of quasi-monoenergetic neutrons generated at $0^{\circ}$ by protons accelerated on a thin LiF target at different energies. The expected neutron energies are also indicated. 
characterized by a resolution of 6.3\% (FWHM) and a detection efficiency of $3.3 \cdot 10^{-7}$ counts per generated neutron.

The ACSpect and the spectrometer developed by Tomita et al., 2010 show similar performance. However, the ACSpect is characterized by a simpler, transportable and more compact layout and does not require a thin $(100 \mu \mathrm{m})$ converter.

\subsection{Continuous neutron spectra}

The ACSpect was irradiated at the LNL with secondary neutrons generated by $4 \mathrm{MeV}$ and $5 \mathrm{MeV}$ protons striking a thick beryllium target. Neutrons are produced on beryllium via the reactions ${ }^{9} \mathrm{Be}(\mathrm{p}, \mathrm{np}) 2 \alpha,{ }^{9} \mathrm{Be}(\mathrm{p}, \mathrm{np}){ }^{8} \mathrm{Be},{ }^{9} \mathrm{Be}(\mathrm{p}, \mathrm{n}){ }^{9} \mathrm{~B}$ and ${ }^{9} \mathrm{Be}(\mathrm{p}, \mathrm{n} \alpha){ }^{5} \mathrm{Li}$ (threshold energy $\mathrm{E}_{\mathrm{th}}=1.75,1.85,2.06$ and $3.93 \mathrm{MeV}$, respectively). The spectrometer was placed at $0^{\circ}$ with respect to the beam direction and at a distance of about $17.5 \mathrm{~mm}$ from the beryllium target. The energy distributions of the neutron yield were compared with those measured by Howard et al., 2001 with the ToF technique. The neutron yield spectra are shown in Fig. 24.

The energy distribution of the yield of neutrons from $4 \mathrm{MeV}$ deuterons on a thick beryllium target was also assessed with the ACSpect and compared to the data measured by Guzek, 1999 with the ToF technique (Fig. 25).

The minimum detectable energy of the ACSpect resulted be about $250 \mathrm{keV}$.

The agreement between the ToF and the ACSpect spectra is
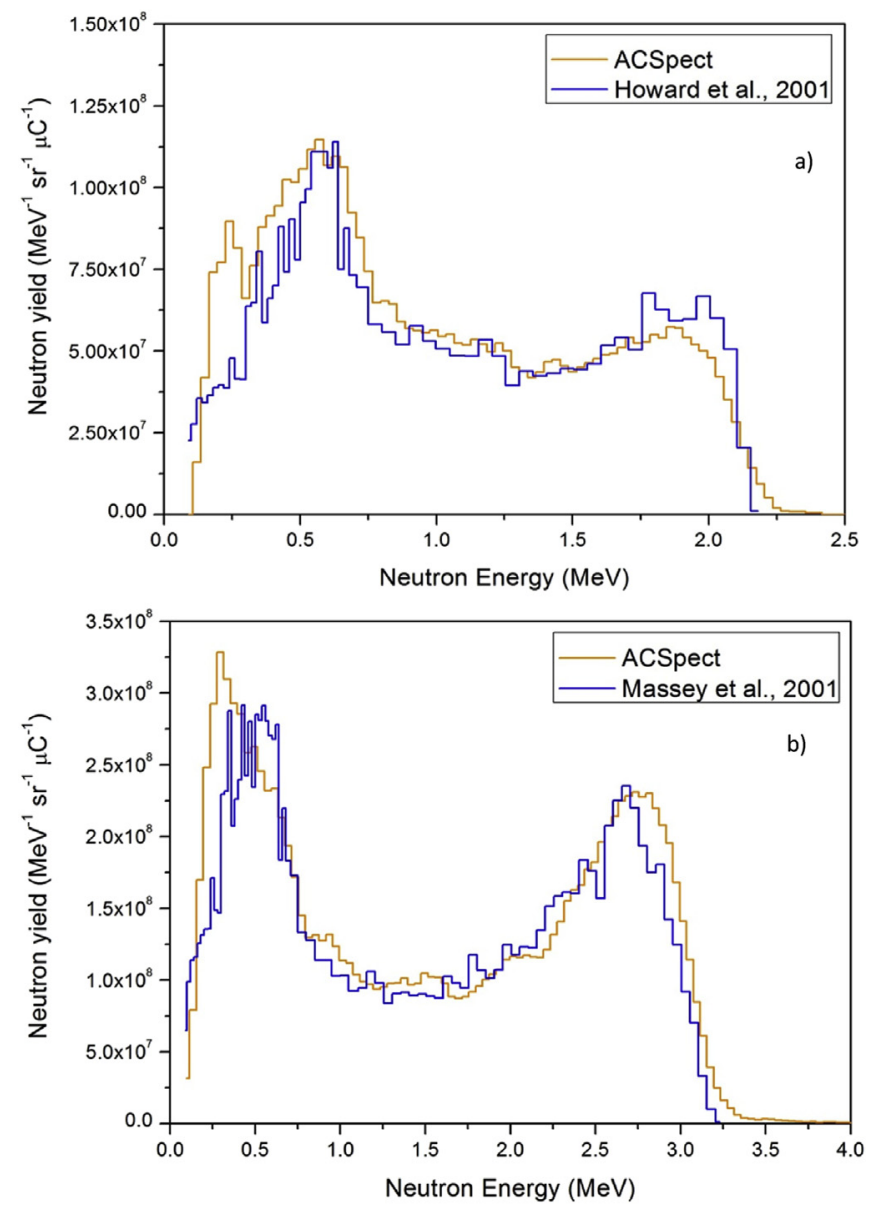

Fig. 24. Energy distribution of the yield of neutrons generated at $0^{\circ}$ by $4 \mathrm{MeV}$ (a) and $5 \mathrm{MeV}$ (b) protons on a thick beryllium target. The spectra measured by Howard et al., 2001 through the TOF technique are also plotted.

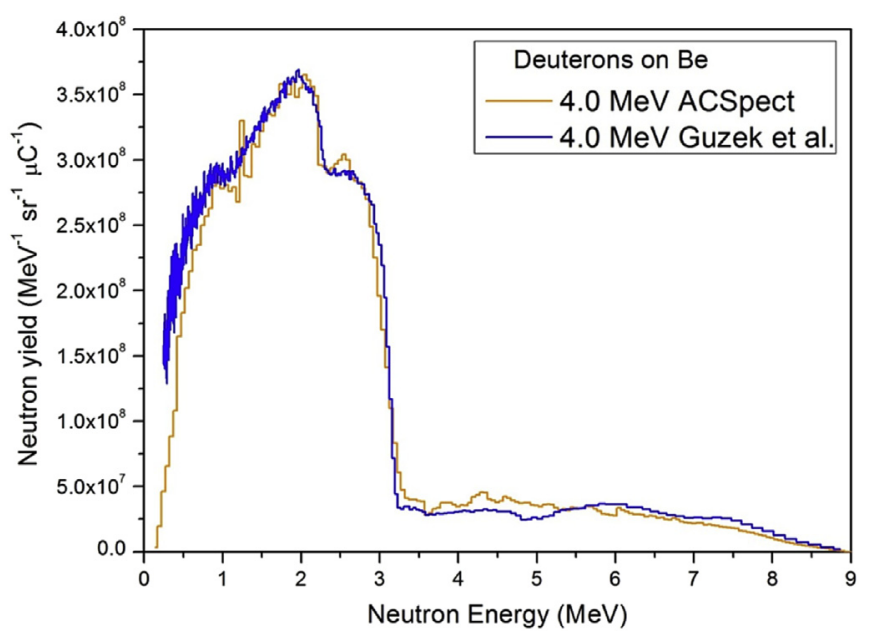

Fig. 25. Energy distribution of the yield of neutrons generated at $0^{\circ}$ by $4 \mathrm{MeV}$ deuterons on a thick beryllium target. The spectrum measured by Guzek, 1999 through the TOF technique is also plotted.

satisfactory for all the considered continuous energy distributions. It should be stressed again that the spectra measured with the ACSpect were acquired directly in real-time.

\section{Conclusions}

A compact neutron spectrometer (ACSpect), based on the recoilproton method, was proposed for direct, real-time and high energy resolution spectrometry of neutron fields.

The basic idea was to use a converter constituted by a plastic scintillator capable of measuring the energy deposited in the converter itself by recoil-protons. A monolithic silicon telescope (MST), measuring the residual proton energy ( $\Delta \mathrm{E}$ stage and $\mathrm{E}$ stage) and discriminating gamma-rays from neutrons, is placed at a proper distance from the scintillator in order to limit the detection to recoil protons emitted at small angles. Event-by-event, the sum of the energy deposited in each stage gives the total energy of the recoilproton and therefore the energy of the impinging neutron (since the emission angle is fixed).

An innovative procedure for event discrimination was developed, by exploiting the MST structure and the time-energy correlation between the three stages, leading to a very accurate selection of recoil-protons. Another procedure was developed to correct the non-linear behavior of the plastic scintillator; by combining the information measured by the PMT and $\mathrm{E}_{\mathrm{MST}}$, the total energy of the recoil-proton can be easily calculated.

The ACSpect is highly directional, i.e. the neutron source should approach a point-source configuration. Therefore it can be applied for measuring the energy distribution of neutrons generated by accelerated charged particles on targets.

The system was tested with monoenergetic neutrons, demonstrating to possess linearity and an energy resolution of $235 \mathrm{keV}$ FWHM at $3.309 \mathrm{MeV}$ (7.1\%). The detection efficiency of the selected geometrical configuration resulted to be of the order of $10^{-6}$ counts per unit neutron fluence, similar to that of spectrometer based on the same principle.

The measurements performed with continuously distributed neutrons generated by protons and deuterons on a beryllium target showed a good agreement with the literature time-of-flight data.

In conclusion, the ACSpect proved to be a simple and compact system, capable of performing direct real-time neutron spectrometry in the $0-6 \mathrm{MeV}$ range with a very satisfactory energy 
resolution.

\section{References}

Agosteo, S., D'Angelo, G., Fazzi, A., Foglio Para, A., Pola, A., Zotto, P., 2007. Neutron spectrometry with a monolithic silicon telescope. Radiat. Prot. Dosim. 126, $210-217$.

Agosteo, S., Pola, A., 2008. Analytical model for a monolithic silicon telescope. Response function of the E Stage. Radiat. Meas. 43, 1487-1492.

Agosteo, S., Colautti, P., Esposito, J., Fazzi, A., Introini, M.V., Pola, A., 2011. Characterization of the energy distribution of neutrons generated by $5 \mathrm{MeV}$ protons on a thick beryllium target at different emission angles. Appl. Radiat. Isot. 69 (12), 1664-1667.

Aleksan, R., Bouchez, J., Cribier, M., Kajfasz, E., Pichard, B., Pierre, F., Poinsignon, J., Spiro, M. Thomas, J.F., 1989. Measurement of fast neutrons in the Gran Sasso laboratory using a ${ }^{6} \mathrm{Li}$ doped liquid scintillator. Nucl. Instrum. Meth. A 274 203-206.

Angelone, M., Pillon, M., Faccini, M., Pinci, D., Baldini, W., Calabrese, R., Cibinetto, G., Cotta Ramusino, A. Malaguti, R., Pozzati, M., 2010. Silicon photo-multiplier radiation hardness tests with a beam controlled neutron source. Nucl. Instrum. Meth. A 623, 921-926.

Aoyama, T., Honda, K., Mori, C., 1993. Energy response of a full-energy-absorption neutron spectrometer using boron-loaded liquid scintillator BC-523. Nucl Instrum. Meth. A 333, 492-501.

Bart Czirr, J., 1994. A compact neutron coincidence spectrometer, its measured response functions and potential applications. Nucl. Instrum. Meth. A 349, 532-539.

Bedogni, R., Bortot, D., Buonomo, B., Esposito, Gómez-Ros, J.M.A., Introini, M.V., Lorenzoli, M., Pola, A., Sacco, D., 2014. First test of SP2: a novel active neutron spectrometer condensing the functionality of Bonner spheres in a single moderator. Nucl. Instrum. Meth. A 767, 159-162.

Bertin, A., Bruschi, M., Bystritsky, V.M., Capponi, M., Cereda, B., D'Antone, I., De Castro, S., Galli, D., Giaobbe, B., Marconi, U., Massa, I., Moroni, C., Piccinini, M., Poli, M., Semprini-Cesari, N., Spighi, R., Stolupin, V.A., Vecchi, S., Vezzani, A. Villa, M., Vitale, A., Wozniak, J., Zavattini, G., Zoccoli, A., 1994. Performances of a coincidence neutron spectrometer with double pulse-shape discrimination. Nucl. Instrum. Meth. A 337, 445-460.

Birks, J.B., 1951. Scintillators from organic crystals: specific fluorescence and relative response to different radiations. Proc. Phys. Soc. A64, 874-877.

Birks, J.B., 1964. The Theory and Practice of Scintillation Counting. Pergamon, London.

Böttger, R., Klein, H., Chalupka, A., Strohmaier, B., 1990. Investigation of the spectral fluence of neutrons from spontaneous fission of ${ }^{252} \mathrm{Cf}$ by means of time-offlight spectrometry. Nucl. Sci. Eng. 106 (3), 377-398.

Bramblett, R.L., Ewing, R.I., Bonner, T.W., 1960. A new type of neutron spectrometer Nucl. Instrum. Meth. 9, 1-12.

Brooks, F.D., Klein, H., 2002. Neutron spectrometry - historical review and present status. Nucl. Instrum. Meth. A 476, 1-11.

Colonna, N., Tagliente, G., 1998. Response of liquid scintillator detectors to neutrons of En $<1 \mathrm{MeV}$. Nucl. Instrum. Meth. A 416, 109-114.

Elevant, T., Van Belle, P., Jarvis, O.N., Sadler, G., 1995. Measurements of fusion neutron energy spectra at JET by means of time-of-flight techniques. Nucl. Instrum. Meth. A 364, 333-341.
Elevant, T., 2002. Fusion neutron energy spectra measured by time-of-flight spectrometers. Nucl. Instrum. Meth. A 476, 485-489.

Ghioni, M., Gulinatti, A., Rech, I., Zappa, F., Cova, S., 2007. Progress in silicon singlephoton avalanche diodes. IEEE J. Sel. Top. Quantum Electron 13 (4), 852-862.

Guzek, J., 1999. Elemental Radiography Using Fast Neutron Beams (PhD. thesis). University of the Witwatersrand, Johannesburg, South Africa.

Harvey, J.A., Hill, N.W., 1979. Scintillation detectors for fast neutron physics. Nucl. Instrum. Meth. 162, 507-530.

Howard, W.B., Grimes, S.M., Massey, T.N., Al-Quraishi, S.I., Jacobs, D.K., Brient, C.E., Yanch, J.C., 2001. Measurement of the thick-target ${ }^{9} \mathrm{Be}(\mathrm{p}, \mathrm{n})$ neutron energy spectra. Nucl. Sci. Eng. 138 (2), 145-160.

ICRU, 1993. Stopping Power and Ranges for Protons and Alpha Particles (Report 49). International Commission on Radiation Units and Measurements, Bethesda, MD.

Ing, H., Clifford, T., McLean, T., Webb, W., Cousins, T., Dhermain, J., 1997. ROSPEC - a simple reliable high resolution neutron spectrometer. Radiat. Prot. Dosim. 70, $273-278$.

Kamykowski, E.A., 1992. Comparison of calculated and measured spectral response and intrinsic efficiency for a boron-loaded plastic neutron detector. Nucl. Instrum. Meth. A 317, 559-566.

Klein, H., Neumann, S., 2002. Neutron and photon spectrometry with liquid scintillation detectors in mixed fields. Nucl. Instrum. Meth. A 476, 132-142.

Knoll, G.F., 2010. Radiation Detection and Measurement, fourth ed. John Wiley and Sons, New York.

Kurosawa, T., Nakamura, T., Nakao, N., Shibata, T., Uwamino, Y., Fukumura, A., 1999. Spectral measurements of neutrons protons, deuterons and tritons produced by $100 \mathrm{MeV} /$ nucleon He bombardment. Nucl. Instrum. Meth. A 430, 400-422.

Manduchi, C., Ciricillo, C., Milli, E., Salviato, S., 1995. Peformance of an isochronous flight path neutron spectrometer. Nucl. Instrum. Meth. A 361, 548-553.

Meigo, S., Takada, H., Chiba, S., Nakamoto, T., Ishibashi, K., Matsufuji, N., Maehata, K. Shigyo, N., Watanabe, Y., Numajiri, M., 1999. Measurements of neutron spectra produced from a thick lead target bombarded with $0.5-$ and $1.5-\mathrm{GeV}$ protons. Nucl. Instrum. Meth. A 431, 521-530.

Pichenot, G, Guldbakke, S., Asselineau, B, Gressier, V., Itié, C, Klein, H., Knauf, K. Lebreton, L., Löb, S., Pochon-Guérin, L., Schlegel, D., Sosaat, W., 2002. Characterisation of spherical recoil proton proportional counters used for neutron spectrometry. Nucl. Instrum. Meth. A 476, 165-169.

Rosenstock, W., Köble, T., Kruzinski, G., Jaunich, G., 1997. Calibration of a neutron spectrometer in the energy range $144 \mathrm{keV}$ to $14.8 \mathrm{MeV}$ with ISO energies. Radiat. Prot. Dosim. 70, 299-303.

Tomita, H., Iwai, H., Iguchi, T., Isobe, M., Kawarabayashi, J., Konno, C., 2010. Development of neutron spectrometer toward deuterium plasma diagnostics in LHD. Rev. Sci. Instrum. 81, 10D309. http://dx.doi.org/10.1063/1.3464260.

Torrisi, L., 2000. Plastic scintillator investigations for relative dosimetry in protontherapy. Nucl. Instrum. Meth. B 523, 161-170.

Tudisco, S., Amorini, F., Cabibbo, M., Cardella, G., De Geronimo, G., Di Pietro, A., Fallica, G., Figuera, P., Musumarra, A., Papa, M., Pappalardo, G., Rizzo, F., Valvo, G., 1999. A new large area monolithic silicon telescope. Nucl. Instrum. Meth. A 426, 436-445.

Zappa, F., Tisa, S., Tosi, A., Cova, S., 2007. Principles and features of single-photon avalanche diode arrays. Sens. Actuators A Phys. 140, 103-112.

Ziegler, J.F., Biersack, J.P., Littmark, U., 1985. In: The Stopping and Range of Ions in Solids. Pergamon Press, New York.

Ziegler, J.F., Biersack, J.P., 2008. In: SRIM 2008. IBM. Available on: www.srim.org. 